JIRSS (2021)

Vol. 20, No. 02, pp 153-181

DOI:10.52547/jirss.20.1.153

\title{
Prediction Based on Type-II Censored Coherent System Lifetime Data under a Proportional Reversed Hazard Rate Model
}

\author{
Adeleh Fallah ${ }^{1}$, Akbar Asgharzadeh ${ }^{1}$, and Hon Keung Tony $\mathrm{Ng}^{2}$ \\ ${ }^{1}$ Department of Statistics, Faculty of Mathematical Sciences, University of Mazandaran, Babolsar, \\ Iran. \\ ${ }^{2}$ Department of Statistical Science, Southern Methodist University, Dallas, Texas 75275-0332, \\ USA.
}

Received: 23/07/2020, Revision received: 23/12/2020, Published online: 03/04/2021

\begin{abstract}
In this paper, we discuss the prediction problem based on censored coherent system lifetime data when the system structure is known and the component lifetime follows the proportional reversed hazard model. Different point and interval predictors based on classical and Bayesian approaches are derived. A numerical example is presented to illustrate the prediction methods used in this paper. Monte Carlo simulation study is performed to evaluate and compare the performances of different prediction methods.
\end{abstract}

Keywords. Bayesian Predictor, Best Unbiased Predictor, Coherent System, Conditional Median Predictor, Maximum Likelihood Predictor, Prediction Intervals.

MSC: 62N05, 90B25.

Adeleh Fallah (adeleh.fallah@gmail.com)

Corresponding Author: Akbar Asgharzadeh (a.asgharzadeh@umz.ac.ir)

Hon Keung Tony Ng (ngh@mail.smu.edu) 


\section{Introduction}

In reliability and system lifetime data analysis, the study of coherent systems is one of the important topics. Researchers and experimenters are interested in learning the lifetime characteristic of the system as well as the lifetime characteristic of the components that make up the system. There are numerous situations that the lifetimes of $k$-component coherent systems can be observed but not the lifetimes of the components (see, for example, $\mathrm{Ng}$ et al., 2012, Yang et al. 2016, 2019) and the prediction of the future failures is of interest. Hence, in this paper, we consider the prediction of future system failures based on Type-II censored system lifetime data.

When the component lifetime follows an absolutely continuous distribution, the failure time of a $k$-component system corresponds to the failure time of one of the $k$ components. Let $T$ be the lifetime of a $k$-component coherent system fromed by $k$ independent and identically distributed (i.i.d.) components in which the lifetimes $X_{1}, X_{2}, \ldots, X_{k}$ follow a distribution with a common absolutely continuous cumulative distribution function $(\mathrm{CDF}) F_{X}(\cdot)$, probability density function $(\mathrm{PDF}) f_{X}(\cdot)$, and survival function (SF) $\bar{F}_{X}(\cdot)=1-F_{X}(\cdot)$. We denote the corresponding order statistics of the lifetimes of the $k$ components as $X_{1: k}<X_{2: k}<\cdots<X_{k: k}$. Furthermore, we denote the SF of the $i$-th order statistic by $\bar{F}_{i: k}(\cdot)$. Suppose $n$ independent $k$-component systems with the same structure are placed on a life-test and the corresponding system lifetimes $T_{1}, T_{2}, \ldots, T_{n}$ are i.i.d. with $\operatorname{CDF}_{T}(\cdot), \operatorname{PDF} g_{T}(\cdot)$ and $\operatorname{SF} \bar{G}_{T}(\cdot)=1-G_{T}(\cdot)$.

To describe the structure of a coherent system, we consider the concept of system signature of a coherent system introduced by Samaniego (1985). Samaniego (1985) defined the system signature $\mathbf{p}=\left(p_{1}, p_{2}, \cdots, p_{k}\right)$ of a coherent system with lifetime $T$ as

$$
p_{j}=\operatorname{Pr}\left(T=X_{j: k}\right),
$$

where the coefficients $p_{1}, p_{2}, \cdots, p_{k}$ are some non-negative real numbers in $[0,1]$ that do not depend on $F_{X}$ and satisfy $\sum_{j=1}^{k} p_{j}=1$. Samaniego (1985) showed that the system signature $\mathbf{p}$ only depends on the structure function of the system but not on the lifetime distribution of the components.

In this study, we assume that the system signature $\mathbf{p}$ of the systems under investigation is known. Samaniego (1985) showed that the PDF and SF of the system lifetime $T$ can be written as (see also, Kochar et al., 1999 and Samaniego, 2007)

$$
g_{T}(t)=\sum_{j=1}^{k} p_{j} f_{j: k}(t) \text { and } \bar{G}_{T}(t)=\sum_{j=1}^{k} p_{j} \bar{F}_{j: k}(t),
$$


respectively, where

$$
f_{j: k}(t)=\left(\begin{array}{c}
k \\
j
\end{array}\right) j f_{X}(t)\left[F_{X}(t)\right]^{j-1}\left[\bar{F}_{X}(t)\right]^{k-j},
$$

and

$$
\bar{F}_{j: k}(t)=\sum_{l=0}^{j-1}\left(\begin{array}{l}
k \\
l
\end{array}\right)\left[F_{X}(t)\right]^{l}\left[\bar{F}_{X}(t)\right]^{k-l},
$$

are respectively the PDF and SF of the $j$-th ordered component lifetime $X_{j: k}$. This representation is called the Samaniego representation. An algorithm to obtain the system signatures of a $k$-component coherent system is proposed by Navarro and Rubio (2010). For instance, the system signatures for five-component coherent systems are presented in Navarro and Rubio (2010).

From Navarro et al. (2007), the reliability function of a coherent system, $\bar{G}_{T}(t)$, can be expressed as

$$
\bar{G}_{T}(t)=\sum_{j=1}^{k} a_{j} \bar{F}_{1: j}(t)=\sum_{j=1}^{k} a_{j}\left[\bar{F}_{X}(t)\right]^{j},
$$

where $a_{1}, a_{2}, \ldots, a_{k}$ are integers (which can be positive or non-positive) that do not depend on the component lifetime distribution and satisfy $\sum_{j=1}^{k} a_{j}=1$, and $\bar{F}_{1: j}(\cdot)$ is the SF of the lifetime of a series system with $j$ components, i.e., $X_{1: j}=\min \left(X_{1}, X_{2}, \ldots, X_{j}\right)$, for $j=1,2, \ldots, k$. The vector $\mathbf{a}=\left(a_{1}, a_{2}, \ldots, a_{k}\right)$ is called the minimal signature of the system (Navarro et al., 2007). For a given system signature $\mathbf{p}$, the corresponding minimal signature a can be obtained. Similarly, for a given minimal signature a, the corresponding system signature $\mathbf{p}$ can be obtained (see, for example, Navarro et al., 2007, 2008).

In recent years, many authors studied the statistical inference of the component lifetime distribution based on system lifetime data when the system signature is known; see, for example, Bhattacharya and Samaniego (2010), Balakrishnan et al. (2011a), Balakrishnan et al. (2011b), Ng et al. (2012), Chahkandi et al. (2014), Zhang et al. (2015a), Zhang et al. (2015b) and Yang et al. (2016, 2019). Although extensive work has been done on parametric and nonparamrtric statistical inference based on system lifetime data with specified system signature, prediction problem with system signature being available has not been studied. Therefore, the aim of this paper is to consider the prediction problem for future failure times of coherent systems with Type-II right-censored experiment when the system signature is known.

In a Type-II censored experiment, $n$ independent $k$-components systems with the 
same system structure are placed on a life-testing experiment and the experiment is terminated when the $m$-th (where $m \leq n$ is pre-fixed) failure is observed. In other words, only the first $m$ failures out of the $n$ systems in the life-test will be observed. The ordered system lifetime data obtained from such a life-test, denote as $T_{1: n}<T_{2: n}<\ldots<T_{m: n}$, is referred to as a Type-II censored sample. Based on the observed Type-II censored system lifetime data, we aim to predict the future system failures $T^{\prime}=T_{s+m: n}(s=1,2, \ldots, n-m)$ when the system signature (or, equivalently, the minimal signature) is available. Under the assumption that the component lifetime is modeled by the proportional reversed hazard rate (PRHR) model, we derive the maximum likelihood predictor, the best unbiased predictor, the conditional median predictor, and the Bayesian point predictor for future system failures $T^{\prime}=T_{s+m: n}(s=1,2, \ldots, n-m)$. Furthermore, we present the prediction intervals (PIs) for future failures $T^{\prime}=T_{s+m: n}(s=1,2, \ldots, n-m)$. To compare the performances of different point and interval prediction methods, a Monte Carlo simulation study is used.

The rest of the paper is organized as follows. Section 2 provides the model of the component and system lifetimes and the maximum likelihood estimator of the exponentiated parameter. In Section 3, we provide different point predictors for the future system failures. Different PIs for the system failures are provided in Section 4. An illustrative example and a Monte Carlo simulation study are presented in Section 5. Finally, some concluding remarks and practical recommendations are provided.

\section{Model and Maximum Likelihood Estimation}

In this paper, it is supposed that the common distribution of the $k$ i.i.d. component lifetimes in a coherent system is the PRHR model with CDF

$$
F_{X}(t)=\left[F_{0}(t)\right]^{\theta}
$$

where $\theta>0$ is the unknown exponentiated (and also reverse proportionality) parameter and $F_{0}(\cdot)$ is the baseline CDF of a lifetime distribution which is completely specified and does not depend on the parameter $\theta$. This family of distributions is also known as the exponentiated family of distributions, since $F_{0}(\cdot)$ is exponentiated by $\theta$. The PRHR model is a flexible model that covers both monotonic and non-monotonic failure rates in many cases.

Some members of the PRHR model which are commonly used in lifetime data analysis are presented as follows: 
(i) Inverse exponential: $\mathbf{I E X P}(\theta)$ with baseline CDF

$$
F_{0}(t)=e^{-\frac{1}{t}}, \quad t>0,
$$

and the CDF is

$$
F_{X}(t)=e^{-\frac{\theta}{t}}, \quad t>0, \quad \theta>0
$$

(ii) Generalized exponential distribution: $\mathrm{GE}(\theta)$ with baseline CDF

$$
F_{0}(t)=1-e^{-t}, \quad t>0
$$

and the $\mathrm{CDF}$ is

$$
F_{X}(t)=\left(1-e^{-t}\right)^{\theta}, \quad t>0, \quad \theta>0 .
$$

(iii) Generalized Rayleigh distribution: $\mathbf{G R}(\theta)$ with baseline CDF

$$
F_{0}(t)=1-e^{-t^{2}}, \quad t>0,
$$

and the CDF is

$$
F_{X}(t)=\left(1-e^{-t^{2}}\right)^{\theta}, \quad t>0, \quad \theta>0 .
$$

(iv) Burr Type III distribution: Burr III $(\theta)$ with baseline CDF

$$
F_{0}(t)=\frac{1}{1+t^{-c}}, \quad t>0, \quad c>0
$$

where the parameter $c$ is assumed to be known, and the CDF is

$$
F_{X}(t)=\left(1+t^{-c}\right)^{-\theta}, \quad t>0, \quad \theta>0 .
$$

(v) Inverse Weibull distribution: $\mathbf{I W}(\theta)$ with baseline CDF

$$
F_{0}(t)=e^{-t^{-\alpha}}, \quad t>0, \quad \alpha>0,
$$

where the parameter $\alpha$ is assumed to be known, and the CDF is

$$
F_{X}(t)=e^{-\theta t^{-\alpha}}, \quad t>0, \quad \theta>0 .
$$


Based on the model in Eq. (2.1) and from Eq. (1.1), the PDF and SF of the system lifetime are given by

$$
g_{T}(t)=\theta \frac{f_{0}(t)}{F_{0}(t)} \sum_{j=1}^{k} j a_{j} F_{0}^{\theta}(t)\left(1-F_{0}^{\theta}(t)\right)^{j-1},
$$

and

$$
\bar{G}_{T}(t)=\sum_{j=1}^{k} a_{j}\left(1-F_{0}^{\theta}(t)\right)^{j},
$$

respectively, where $f_{0}(t)=d F_{0}(t) / d t$ is the baseline PDF.

Suppose $T_{1: n}<T_{2: n}<\cdots<T_{m: n}$ is an ordered Type II censored sample from a population with PDF $g_{T}(t ; \theta)$ and $\operatorname{CDF}_{T}(t ; \theta)$ in Eqs. (2.2) and (2.3). For notation simplicity, we denote the observed values of $T_{1: n}<T_{2: n}<\cdots<T_{m: n}$ by $t_{1}<t_{2}<\cdots<t_{m}$ instead of $t_{1: n}<t_{2: n}<\cdots<t_{m: n}$. The joint PDF of $\mathbf{T}=\left(T_{1: n}, T_{2: n}, \ldots, T_{m: n}\right)$ is given by

$$
L(\theta \mid \mathbf{t})=\frac{n !}{(n-m) !} \prod_{i=1}^{m} g_{T}\left(t_{i} ; \theta\right)\left[1-G_{T}\left(t_{m} ; \theta\right)\right]^{n-m},
$$

where $\mathbf{t}=\left(t_{1}, t_{2}, \ldots, t_{m}\right)$ is the vector of observations. From Eqs. (2.2), (2.3) and (2.4), the log-likelihood function can be expressed as

$$
\begin{aligned}
\log L(\theta \mid \mathbf{t})= & \log C+m \log \theta+\theta \sum_{i=1}^{m} \log F_{0}\left(t_{i}\right)+\sum_{i=1}^{m} \log \left\{\sum_{j=1}^{k} j a_{j}\left(1-F_{0}^{\theta}\left(t_{i}\right)\right)^{j-1}\right\} \\
& +(n-m) \log \left\{\sum_{j=1}^{k} a_{j}\left(1-F_{0}^{\theta}\left(t_{m}\right)\right)^{j}\right\},
\end{aligned}
$$

where $C=\frac{n !}{(n-m) !} \prod_{i=1}^{m} \frac{f_{0}\left(t_{i}\right)}{F_{0}\left(t_{i}\right)}$ is a constant that is independent of the parameter $\theta$. Then, we can obtain the likelihood equation as

$$
\begin{aligned}
\frac{d \log L(\theta \mid \mathbf{t})}{d \theta}= & \frac{m}{\theta}+\sum_{i=1}^{m} \log F_{0}\left(t_{i}\right)-\sum_{i=1}^{m} \log F_{0}\left(t_{i}\right)\left(\frac{\sum_{j=1}^{k} j(j-1) a_{j} F_{0}^{\theta}\left(t_{i}\right)\left(1-F_{0}^{\theta}\left(t_{i}\right)\right)^{j-2}}{\sum_{j=1}^{k} j a_{j}\left(1-F_{0}^{\theta}\left(t_{i}\right)\right)^{j-1}}\right) \\
& -(n-m) \log F_{0}\left(t_{m}\right)\left(\frac{\sum_{j=1}^{k} j a_{j} F_{0}^{\theta}\left(t_{m}\right)\left(1-F_{0}^{\theta}\left(t_{m}\right)\right)^{j-1}}{\sum_{j=1}^{k} a_{j}\left(1-F_{0}^{\theta}\left(t_{m}\right)\right)^{j}}\right)=0 .
\end{aligned}
$$


Therefore, the maximum likelihood estimator (MLE) of $\theta$, denoted as $\hat{\theta}_{M L E}$ can be obtained by solving Eq. (2.6) with respect to $\theta$. Since the likelihood equation is a non-linear equation, therefore, the MLE of $\theta$ needs to be obtained by using numerical methods.

\section{Point Predictors}

Because of the Markov property of the conditional order statistics, the conditional distribution of $T^{\prime}=T_{s+m: n}(s=1,2, \ldots, n-m)$, given $\mathbf{T}=\mathbf{t}=\left(t_{1}, t_{2}, \ldots, t_{m}\right)$ is equal to the conditional distribution of $T^{\prime}$ given $T_{m: n}=t_{m}$. As a result, the conditional PDF of $T^{\prime}$ given $T_{m: n}=t_{m}$ is the same as the PDF of the $s$-th order statistic from a sample of size $n-m$ from the population with PDF $g\left(t^{\prime} ; \theta\right) /\left[1-G\left(t_{m} ; \theta\right)\right], t^{\prime} \geq t_{m}$ (i.e., a left-truncated PDF with truncation point $t_{m}$ ). Therefore, the conditional PDF of $T^{\prime}$ given $T_{m: n}=t_{m}$ is

$$
\begin{aligned}
h\left(t^{\prime} \mid t_{m} ; \theta\right)= & s\left(\begin{array}{c}
n-m \\
s
\end{array}\right) g_{T}\left(t^{\prime} ; \theta\right)\left[G_{T}\left(t^{\prime} ; \theta\right)-G_{T}\left(t_{m} ; \theta\right)\right]^{s-1} \\
& \times\left[1-G_{T}\left(t^{\prime} ; \theta\right)\right]^{n-m-s}\left[1-G_{T}\left(t_{m} ; \theta\right)\right]^{-(n-m)}, t^{\prime} \geq t_{m} .
\end{aligned}
$$

For the PDF and SF of the system lifetime presented in Eqs. (2.2) and (2.3), the conditional PDF in Eq. (3.1), for $t^{\prime} \geq t_{m}$, reduces to

$$
\begin{aligned}
h\left(t^{\prime} \mid \mathbf{t} ; \theta\right)= & s\left(\begin{array}{c}
n-m \\
s
\end{array}\right) \theta \frac{f_{0}\left(t^{\prime}\right)}{\left[F_{0}\left(t^{\prime}\right)\right]^{1-\theta}} \times\left(\sum_{j=1}^{k} j a_{j}\left(1-F_{0}^{\theta}\left(t^{\prime}\right)\right)^{j-1}\right)\left(\sum_{j=1}^{k} a_{j}\left(1-F_{0}^{\theta}\left(t^{\prime}\right)\right)^{j}\right)^{n-m-s} \\
& \times\left[\sum_{j=1}^{k} a_{j}\left(1-F_{0}^{\theta}\left(t_{m}\right)\right)^{j}-\sum_{j=1}^{k} a_{j}\left(1-F_{0}^{\theta}\left(t^{\prime}\right)\right)^{j}\right]^{s-1} \\
& \times\left(\sum_{j=1}^{k} a_{j}\left(1-F_{0}^{\theta}\left(t_{m}\right)\right)^{j}\right)^{-(n-m)} .
\end{aligned}
$$

Several different point predictors for $T^{\prime}=T_{s+m: n}(s=1,2, \ldots, n-m)$ are discussed in the following subsection.

\subsection{Maximum Likelihood Predictor}

In this subsection, the likelihood approach is used to obtain the maximum likelihood predictor (MLP) for $T^{\prime}=T_{s+m: n}(s=1,2, \ldots, n-m)$. The likelihood approach, introduced 
by Kaminsky and Rodhin (1985), has become a very useful tool to estimate the parameters involved in the model and to predict the future order statistics simultaneously; see, e.g., Basak et al. (2006), Basak and Balakrishnan (2017), Asgharzadeh et al. (2015, 2018), Raqab et al. (2019) and Saadati Nik et al. (2020). Based on the observed Type-II censored sample $\mathbf{t}=\left(t_{1}, \ldots, t_{m}\right)$, the predictive likelihood function (PLF) of $T^{\prime}$ and $\theta$ is considered and maximized with respect to the future observations $T^{\prime}$ and the unknown parameter $\theta$ simultaneously. The PLF of $T^{\prime}$ and $\theta$, is given by

$$
L\left(T^{\prime}, \theta \mid \mathbf{t}\right)=h\left(t^{\prime} \mid \mathbf{t} ; \theta\right) L(\mathbf{t} ; \theta)
$$

Suppose $\widehat{T^{\prime}}=u(\mathbf{T})$ and $\widehat{\theta}=v(\mathbf{T})$ are statistics for which

$$
L(u(\mathbf{t}), v(\mathbf{t}) \mid \mathbf{t})=\sup _{\left(t^{\prime}, \theta\right)} L\left(t^{\prime}, \theta \mid \mathbf{t}\right)
$$

where $u(\mathbf{t})$ and $v(\mathbf{t})$ are the values of the statistics $\widehat{T^{\prime}}=u(\mathbf{T})$ and $\widehat{\theta}=v(\mathbf{T})$, respectively, computed based on the observed data $\mathbf{t}$. Then, $u(\mathbf{T})$ is the MLP of $T^{\prime}$ and $v(\mathbf{T})$ is the predictive maximum likelihood estimator (PMLE) of $\theta$. By using Eq. (2.5) and the logarithm of (3.2), the log-PLF of $T^{\prime}$ and $\theta$ can be expressed as

$$
\begin{aligned}
\log L\left(t^{\prime}, \theta \mid \mathbf{t}\right)= & \text { constant }+(m+1) \log \theta+\log f_{0}\left(t^{\prime}\right)-\log F_{0}\left(t^{\prime}\right)+\theta \log F_{0}\left(t^{\prime}\right) \\
& +\theta \sum_{i=1}^{m} \log F_{0}\left(t_{i}\right)+\sum_{i=1}^{m} \log \left(\sum_{j=1}^{k} j a_{j}\left(1-F_{0}^{\theta}\left(t_{i}\right)\right)^{j-1}\right) \\
& +\log \left(\sum_{j=1}^{k} j a_{j}\left(1-F_{0}^{\theta}\left(t^{\prime}\right)\right)^{j-1}\right)+(n-m-s) \log \left(\sum_{j=1}^{k} a_{j}\left(1-F_{0}^{\theta}\left(t^{\prime}\right)\right)^{j}\right) \\
& +(s-1) \log \left(\sum_{j=1}^{k} a_{j}\left(1-F_{0}^{\theta}\left(t_{m}\right)\right)^{j}-\sum_{j=1}^{k} a_{j}\left(1-F_{0}^{\theta}\left(t^{\prime}\right)\right)^{j}\right) .
\end{aligned}
$$

By taking the first derivatives of the predictive log-likelihood function in Eq. (3.4) with respect to $t^{\prime}$ and $\theta$, the predictive likelihood equations (PLEs) for $T^{\prime}=T_{s+m: n}(s=$ 
$1,2, \ldots, n-m)$ and $\theta$ can be obtained as:

$$
\begin{aligned}
\frac{\partial \log L\left(t^{\prime}, \theta \mid \mathbf{t}\right)}{\partial t^{\prime}}= & \frac{f_{0}^{\prime}\left(t^{\prime}\right) F_{0}\left(t^{\prime}\right)+(\theta-1) f_{0}^{2}\left(t^{\prime}\right)}{f_{0}\left(t^{\prime}\right) F_{0}\left(t^{\prime}\right)} \\
& -\left(\frac{\sum_{j=1}^{k} j(j-1) a_{j} \theta f_{0}\left(t^{\prime}\right) F_{0}^{\theta-1}\left(t^{\prime}\right)\left(1-F_{0}^{\theta}\left(t^{\prime}\right)\right)^{j-2}}{\sum_{j=1}^{k} j a_{j}\left(1-F_{0}^{\theta}\left(t^{\prime}\right)\right)^{j-1}}\right) \\
& -(n-m-s)\left(\frac{\sum_{j=1}^{k} j a_{j} \theta f_{0}\left(t^{\prime}\right) F_{0}^{\theta-1}\left(t^{\prime}\right)\left(1-F_{0}^{\theta}\left(t^{\prime}\right)\right)^{j-1}}{\sum_{j=1}^{k} a_{j}\left(1-F_{0}^{\theta}\left(t^{\prime}\right)\right)^{j}}\right) \\
& +(s-1)\left(\frac{\sum_{j=1}^{k} j a_{j} \theta f_{0}\left(t^{\prime}\right) F_{0}^{\theta-1}\left(t^{\prime}\right)\left(1-F_{0}^{\theta}\left(t^{\prime}\right)\right)^{j-1}}{\sum_{j=1}^{k} a_{j}\left(1-F_{0}^{\theta}\left(t_{m}\right)\right)^{j}-\sum_{j=1}^{k} a_{j}\left(1-F_{0}^{\theta}\left(t^{\prime}\right)\right)^{j}}\right)=0,
\end{aligned}
$$

and

$$
\begin{aligned}
\frac{\partial \ln L\left(t^{\prime}, \theta \mid \mathbf{t}\right)}{\partial \theta}= & \frac{m+1}{\theta}+\sum_{i=1}^{m} \log F_{0}\left(t_{i}\right)+\log F_{0}\left(t^{\prime}\right) \\
& -\sum_{i=1}^{m} \log F_{0}\left(t_{i}\right)\left(\frac{\sum_{j=1}^{k} j(j-1) a_{j} F_{0}^{\theta}\left(t_{i}\right)\left(1-F_{0}^{\theta}\left(t_{i}\right)\right)^{j-2}}{\sum_{j=1}^{k} j a_{j}\left(1-F_{0}^{\theta}\left(t_{i}\right)\right)^{j-1}}\right) \\
& -\log F_{0}\left(t^{\prime}\right)\left(\frac{\sum_{j=1}^{k} j(j-1) a_{j} F_{0}^{\theta}\left(t^{\prime}\right)\left(1-F_{0}^{\theta}\left(t^{\prime}\right)\right)^{j-2}}{\sum_{j=1}^{k} j a_{j}\left(1-F_{0}^{\theta}\left(t^{\prime}\right)\right)^{j-1}}\right) \\
& -(n-m-s) \log F_{0}\left(t^{\prime}\right)\left(\frac{\sum_{j=1}^{k} j a_{j} F_{0}^{\theta}\left(t^{\prime}\right)\left(1-F_{0}^{\theta}\left(t^{\prime}\right)\right)^{j-1}}{\sum_{j=1}^{k} a_{j}\left(1-F_{0}^{\theta}\left(t^{\prime}\right)\right)^{j}}\right) \\
& -(s-1)\left\{\frac{\sum_{j=1}^{k} j a_{j} F_{0}^{\theta}\left(t_{m}\right) \log F_{0}\left(t_{m}\right)\left(1-F_{0}^{\theta}\left(t_{m}\right)\right)^{j-1}}{\sum_{j=1}^{k} a_{j}\left(1-F_{0}^{\theta}\left(t_{m}\right)\right)^{j}-\sum_{j=1}^{k} a_{j}\left(1-F_{0}^{\theta}\left(t^{\prime}\right)\right)^{j}}\right. \\
& -\frac{\sum_{j=1}^{k} j a_{j} F_{0}^{\theta}\left(t^{\prime}\right) \log F_{0}\left(t^{\prime}\right)\left(1-F_{0}^{\theta}\left(t^{\prime}\right)\right)^{j-1}}{\sum_{j=1}^{k} a_{j}\left(1-F_{0}^{\theta}\left(t_{m}\right)\right)^{j}-\sum_{j=1}^{k} a_{j}\left(1-F_{0}^{\theta}\left(t^{\prime}\right)\right)^{j}}=0 .
\end{aligned}
$$

By solving Eqs. (3.5) and (3.6) with respect to $t^{\prime}$ and $\theta$ simultaneously, the MLP of 
$T^{\prime}$, denoted as $\widehat{T^{\prime}} M L P$, and the PMLE of $\theta$ can be obtained. Numerical methods can be used to solve Eqs. (3.5) and (3.6).

\subsection{Conditional Predictors}

Consider the statistic $\widehat{T^{\prime}}$ for predicting $T^{\prime}=T_{s+m: n}$, if the prediction error $\left(\widehat{T^{\prime}}-T^{\prime}\right)$ has a mean zero, then the statistic $\widehat{T^{\prime}}$ is called an unbiased predictor of $T^{\prime}$. Furthermore, if its predictor error variance $\operatorname{Var}\left(\widehat{T^{\prime}}-T^{\prime}\right)$ is smaller than or equal to that of any other unbiased predictor of $T^{\prime}$, then the statistic $\widehat{T}^{\prime}$ is called the best unbiased predictor (BUP) of $T^{\prime}$. The BUP of $T^{\prime}$ is the mean of conditional distribution of $T^{\prime}$ given $\mathbf{T}=\mathbf{t}$. Therefore, the BUP of $T^{\prime}$ is given by

$$
\widehat{T}_{B U P}=E_{\theta}\left(T^{\prime} \mid \mathbf{t}\right)=E_{\theta}\left(T^{\prime} \mid t_{m}\right) .
$$

Using Eq. (3.2) and the binomial expansion, we have

$$
\begin{aligned}
& {\left[\sum_{j=1}^{k} a_{j}\left(1-F_{0}^{\theta}\left(t_{m}\right)\right)^{j}-\sum_{j=1}^{k} a_{j}\left(1-F_{0}^{\theta}\left(t^{\prime}\right)\right)^{j}\right]^{s-1} } \\
= & \sum_{l=0}^{s-1}\left[\left(\begin{array}{c}
s-1 \\
l
\end{array}\right)(-1)^{l}\left(\sum_{j=1}^{k} a_{j}\left(1-F_{0}^{\theta}\left(t^{\prime}\right)\right)^{j}\right)^{l}\left(\sum_{j=1}^{k} a_{j}\left(1-F_{0}^{\theta}\left(t_{m}\right)\right)^{j}\right)^{s-l-1}\right] .
\end{aligned}
$$

The conditional PDF of $T^{\prime}$ given $T_{m: n}=t_{m}$ is given by

$$
\begin{aligned}
h\left(t^{\prime} \mid t_{m} ; \theta\right)= & s\left(\begin{array}{c}
n-m \\
s
\end{array}\right) \theta \frac{f_{0}\left(t^{\prime}\right)}{\left[F_{0}\left(t^{\prime}\right)\right]^{1-\theta}}\left(\sum_{j=1}^{k} j a_{j}\left(1-F_{0}^{\theta}\left(t^{\prime}\right)\right)^{j-1}\right) \\
& \times \sum_{l=0}^{s-1}\left\{\left(\begin{array}{c}
s-1 \\
l
\end{array}\right)(-1)^{l}\left(\sum_{j=1}^{k} a_{j}\left(1-F_{0}^{\theta}\left(t^{\prime}\right)\right)^{j}\right)^{n-m-s+l}\right. \\
& \left.\left(\sum_{j=1}^{k} a_{j}\left(1-F_{0}^{\theta}\left(t_{m}\right)\right)^{j}\right)^{s-l-1-n+m}\right\},
\end{aligned}
$$


for $t^{\prime}>t_{m}$. Using Eqs. (3.7) and (3.8), the BUP of $T^{\prime}$ can be obtained as

$$
\begin{aligned}
\widehat{T^{\prime}} \text { BUP }= & \int_{t_{m}}^{\infty} t^{\prime} h\left(t^{\prime} \mid t_{m} ; \theta\right) d t^{\prime} \\
= & s\left(\begin{array}{c}
n-m \\
s
\end{array}\right) \theta\left\{\sum_{l=0}^{s-1}\left(\begin{array}{c}
s-1 \\
l
\end{array}\right)(-1)^{l}\left(\sum_{j=1}^{k} a_{j}\left(1-F_{0}^{\theta}\left(t_{m}\right)\right)^{j}\right)^{s-l-1-n+m}\right. \\
& \left.\int_{t_{m}}^{\infty} \frac{t^{\prime} f_{0}\left(t^{\prime}\right)}{\left[F_{0}\left(t^{\prime}\right)\right]^{1-\theta}}\left(\sum_{j=1}^{k} j a_{j}\left(1-F_{0}^{\theta}\left(t^{\prime}\right)\right)^{j-1}\right)\left(\sum_{j=1}^{k} a_{j}\left(1-F_{0}^{\theta}\left(t^{\prime}\right)\right)^{j}\right)^{n-m-s+l} d t^{\prime}\right\} .
\end{aligned}
$$

When the parameter $\theta$ is unknown, one can approximate the BUP of $T^{\prime}$ by replacing $\theta$ with its corresponding MLE.

Another conditional predictor is the conditional median predictor (CMP). This predictor was first proposed by Raqab and Nagaraja (1995) in the context of order statistics. Consider a predictor $\widehat{T}^{\prime}$ for $T^{\prime}$, if $\widehat{T^{\prime}}$ is the median of the conditional distribution of $T^{\prime}$ given $T_{m: n}=t_{m}$, i.e.,

$$
\operatorname{Pr}_{\theta}\left(T^{\prime} \leq \widehat{T^{\prime}} \mid T_{m: n}=t_{m}\right)=\operatorname{Pr}_{\theta}\left(T^{\prime} \geq \widehat{T^{\prime}} \mid T_{m: n}=t_{m}\right),
$$

then the predictor $\widehat{T^{\prime}}$ is called a CMP of $T^{\prime}$. For the PDF and SF of the system lifetime presented in Eqs. (2.2) and (2.3), we can obtain

$$
\begin{aligned}
& \operatorname{Pr}_{\theta}\left(T^{\prime} \leq \widehat{T^{\prime}} \mid T_{m: n}=t_{m}\right) \\
= & \operatorname{Pr}_{\theta}\left(\frac{G_{T}\left(T^{\prime}\right)-G_{T}\left(t_{m}\right)}{1-G_{T}\left(t_{m}\right)} \leq \frac{G_{T}\left(\widehat{T^{\prime}}\right)-G_{T}\left(t_{m}\right)}{1-G_{T}\left(t_{m}\right)} \mid T_{m: n}=t_{m}\right) \\
= & \operatorname{Pr}_{\theta}\left(1-\frac{\sum_{j=1}^{k} a_{j}\left(1-F_{0}^{\theta}\left(t^{\prime}\right)\right)^{j}}{\sum_{j=1}^{k} a_{j}\left(1-F_{0}^{\theta}\left(t_{m}\right)\right)^{j}} \leq 1-\frac{\sum_{j=1}^{k} a_{j}\left(1-F_{0}^{\theta}\left(\widehat{T^{\prime}}\right)\right)^{j}}{\sum_{j=1}^{k} a_{j}\left(1-F_{0}^{\theta}\left(t_{m}\right)\right)^{j}} \mid T_{m: n}=t_{m}\right) .
\end{aligned}
$$

By using the conditional PDF of $T^{\prime}$ given $T_{m: n}=t_{m}$ in Eq.(3.1), the conditional distribution

$$
\frac{G_{T}\left(T^{\prime}\right)-G_{T}\left(t_{m}\right)}{1-G_{T}\left(t_{m}\right)}=1-\frac{\sum_{j=1}^{k} a_{j}\left(1-F_{0}^{\theta}\left(T^{\prime}\right)\right)^{j}}{\sum_{j=1}^{k} a_{j}\left(1-F_{0}^{\theta}\left(t_{m}\right)\right)^{j}} .
$$


follows a beta distribution with parameters $s$ and $n-m-s+1$ (denote as $\operatorname{Beta}(s, n-m-$ $s+1)$ ). Therefore, by Eq. (3.11), the CMP of $T^{\prime}$ can be obtained by solving

$$
1-\frac{\sum_{j=1}^{k} a_{j}\left(1-F_{0}^{\theta}\left(\widehat{T^{\prime}}\right)\right)^{j}}{\sum_{j=1}^{k} a_{j}\left(1-F_{0}^{\theta}\left(t_{m}\right)\right)^{j}}=\operatorname{Med}(B)
$$

where $B$ is a random variable follows $\operatorname{Beta}(s, n-m-s+1)$ distribution and $\operatorname{Med}(B)$ is the median of random variable $B$. From Eq. (3.12), the CMP of $T^{\prime}, \widehat{T}^{\prime} C M P$, can be computed by solving the nonlinear equation

$$
\left(\sum_{j=1}^{k} a_{j}\left(1-F_{0}^{\theta}\left(t_{m}\right)\right)^{j}\right)(1-\operatorname{Med}(B))-\left(\sum_{j=1}^{k} a_{j}\left(1-F_{0}^{\theta}\left(\widehat{T}^{\prime}\right)\right)^{j}\right)=0 .
$$

When $\theta$ is unknown, we can substitute $\theta$ with its MLE and derive an approximation of the CMP of $T^{\prime}$.

\subsection{Bayesian Point Predictor}

In this subsection, we consider the Bayesian point prediction for the future system failures $T^{\prime}=T_{s+m: n},(s=1,2, \ldots, n-m)$, based on the observed Type-II censored sample $\mathbf{t}=\left(t_{1}, t_{2}, \cdots, t_{m}\right)$. To the ease of mathematical manipulation of the posterior distribution, it is assumed that the exponentiated parameter $\theta$ in PRHR model has a gamma prior distribution with parameters $\alpha$ and $\beta$ (denoted as $\Gamma(\alpha, \beta)$ ) with PDF

$$
\pi(\theta)=\frac{\beta^{\alpha}}{\Gamma(\alpha)} \theta^{\alpha-1} e^{-\beta \theta}, \theta>0, \beta>0, \alpha>0 .
$$

Note that Jeffrey's prior can be obtained as a special case of Eq. (3.14) by taking $\alpha=\beta=0$. Combining Eqs. (2.5) and (3.14), the posterior PDF of $\theta$ given the data is

$$
\pi(\theta \mid \mathbf{t}) \propto \theta^{m+\alpha-1} e^{-\beta \theta} \prod_{i=1}^{m} F_{0}^{\theta}\left(t_{i}\right) \prod_{i=1}^{m}\left\{\sum_{j=1}^{k} j a_{j}\left(1-F_{0}^{\theta}\left(t_{i}\right)\right)^{j-1}\right\}\left\{\sum_{j=1}^{k} a_{j}\left(1-F_{0}^{\theta}\left(t_{m}\right)\right)^{j}\right\}^{n-m} .
$$

For the Bayesian prediction of $T^{\prime}=T_{s+m: n},(s=1,2, \ldots, n-m)$, we first obtain the Bayesian predictive PDF of $T^{\prime}$ given $T_{m}=t_{m}$. This Bayesian predictive PDF at any point $t^{\prime}\left(t^{\prime}>t_{m}\right)$ is

$$
h^{*}\left(t^{\prime} \mid t_{m}\right)=\int_{0}^{\infty} h\left(t^{\prime} \mid t_{m} ; \theta\right) \pi(\theta \mid t) d \theta
$$


By substituting Eqs. (3.8) and (3.15) into Eq. (3.16), the Bayesian predictive PDF, for $t^{\prime}>t_{m}$, is

$$
\begin{aligned}
h^{*}\left(t^{\prime} \mid t_{m}\right)= & \int_{0}^{\infty} s\left(\begin{array}{c}
n-m \\
s
\end{array}\right) \theta^{m+\alpha} e^{-\beta \theta} \frac{f_{0}\left(t^{\prime}\right)}{\left[F_{0}\left(t^{\prime}\right)\right]^{1-\theta}} \prod_{i=1}^{m} F_{0}^{\theta}\left(t_{i}\right) \\
& \times\left(\sum_{j=1}^{k} j a_{j}\left(1-F_{0}^{\theta}\left(t^{\prime}\right)\right)^{j-1}\right) \prod_{i=1}^{m}\left(\sum_{j=1}^{k} j a_{j}\left(1-F_{0}^{\theta}\left(t_{i}\right)\right)^{j-1}\right) \\
& \times \sum_{l=0}^{s-1}\left(\begin{array}{c}
s-1 \\
l
\end{array}\right)(-1)^{l}\left(\sum_{j=1}^{k} a_{j}\left(1-F_{0}^{\theta}\left(t^{\prime}\right)\right)^{j}\right)^{n-m-s+l} \\
& \times\left(\sum_{j=1}^{k} a_{j}\left(1-F_{0}^{\theta}\left(t_{m}\right)\right)^{j}\right)^{s-l-1} d \theta .
\end{aligned}
$$

Therefore, the Bayesian point predictor of $T^{\prime}=T_{s+m: n}(s=1,2, \ldots, n-m)$, under the squared error loss, can be obtained as

$$
T_{\text {Bayes }}^{\prime}=\int_{t_{m}}^{\infty} t^{\prime} h^{*}\left(t^{\prime} \mid t_{m}\right) d t^{\prime} .
$$

Due to the complicated form of $h^{*}\left(t^{\prime} \mid t_{m}\right)$, the Bayesian point predictor in Eq. (3.17) cannot be computed explicitly. Here, we propose using the Metropolis-Hastings algorithm (see, for example, Roberts and Casella, 2004) with Gaussian (normal) proposal distribution to find a simulation-based consistent estimator of $h^{*}\left(t^{\prime} \mid t_{m}\right)$. For our situation, we first generate a Monte Carlo (MC) sample of size $N,\left(\theta_{1}, \theta_{2}, \ldots, \theta_{N}\right)$, from $\pi(\theta \mid \mathbf{t})$ using the Metropolis-Hastings algorithm. Then, by using Eq. (3.16), a simulation-based consistent estimator of $h^{*}\left(t^{\prime} \mid t_{m}\right)$ can be obtained as

$$
\widehat{h^{*}}\left(t^{\prime} \mid t_{m}\right)=\frac{1}{N} \sum_{i=1}^{N} h\left(t^{\prime} \mid t_{m} ; \theta_{i}\right) .
$$

Hence, by using Eqs. (3.17) and (3.18), the Bayesian point predictor can be approximated as

$$
\widehat{T}_{\text {Bayes }}=\int_{t_{m}}^{\infty} t^{\prime} \widehat{h}^{*}\left(t^{\prime} \mid t_{m}\right) d t^{\prime}=\frac{1}{N} \sum_{i=1}^{N} \int_{t_{m}}^{\infty} t^{\prime} h\left(t^{\prime} \mid t_{m} ; \theta_{i}\right) d t^{\prime},
$$

where $h\left(t^{\prime} \mid t_{m} ; \theta_{i}\right)$ is given in Eq. (3.8) with $\theta=\theta_{i}$. The Metropolis-Hastings algorithm for generating the MC sample of size $N$ from $\pi(\theta \mid \mathbf{t})$ is described as follows: 
Step A1. Provide an initial guess $\theta^{(0)}$ of $\theta$;

Step A2. Set $v=1$;

Step A3. Based on the Metropolis-Hastings algorithm, generate $\theta^{(v)}$ from $\pi\left(\theta^{(v-1)} \mid \mathbf{t}\right)$ using the Gaussian distribution with mean $\theta^{(v-1)}$ and variance $S_{\theta}^{2}\left(\right.$ i.e., $\left.N\left(\theta^{(v-1)}, S_{\theta}^{2}\right)\right)$ as the proposal distribution, where $S_{\theta}^{2}$ can be obtained as the inverse of the Fisher information;

Step A4. Set $v=v+1$;

Step A5. Repeat Steps A3 and A4 $N$ times to obtain the sample $\theta_{1}, \theta_{2}, \ldots, \theta_{N}$.

\subsection{Illustration with Inverse Exponential Distribution}

In this subsection, the prediction methods discussed in Sections 3.1-3.3 are illustrated by considering inverse exponential distributed components with baseline $\operatorname{CDF} F_{0}(t)=$ $e^{-\frac{1}{t}}, t>0$.

Maximum likelihood predictor: For $k$-component systems with inverse exponential distributed components, the MLP for $T^{\prime}=T_{s+m: n}(s=1,2, \ldots, n-m)$ and the PMLE of $\theta$ can be computed by solving the Eqs. (3.5) and (3.6) which can be expressed as

$$
\begin{aligned}
\frac{\partial \log L\left(t^{\prime}, \theta \mid \mathbf{t}\right)}{\partial t^{\prime}}= & \frac{-2 t^{\prime}+\theta}{t^{\prime 2}}-\frac{\theta}{t^{\prime 2}} e^{-\theta / t^{\prime}}\left(\frac{\sum_{j=1}^{k} j(j-1) a_{j}\left(1-e^{-\theta / t^{\prime}}\right)^{j-2}}{\sum_{j=1}^{k} j a_{j}\left(1-e^{-\theta / t^{\prime}}\right)^{j-1}}\right) \\
& -(n-m-s) \frac{\theta}{t^{\prime 2}} e^{-\theta / t^{\prime}}\left(\frac{\sum_{j=1}^{k} j a_{j}\left(1-e^{-\theta / t^{\prime}}\right)^{j-1}}{\sum_{j=1}^{k} a_{j}\left(1-e^{-\theta / t^{\prime}}\right)^{j}}\right) \\
& +(s-1) \frac{\theta}{t^{\prime 2}} e^{-\theta / t^{\prime}}\left(\frac{\sum_{j=1}^{k} j a_{j}\left(1-e^{-\theta / t^{\prime}}\right)^{j-1}}{\sum_{j=1}^{k} a_{j}\left(1-e^{-\theta / t_{m}}\right)^{j}-\sum_{j=1}^{k} a_{j}\left(1-e^{-\theta / t^{\prime}}\right)^{j}}\right)=0,
\end{aligned}
$$


and

$$
\begin{aligned}
& \frac{\partial \log L\left(t^{\prime}, \theta \mid \mathbf{t}\right)}{\partial \theta}=\frac{m+1}{\theta}-\frac{1}{t^{\prime}}-\sum_{i=1}^{m} \frac{1}{t_{i}}+\sum_{i=1}^{m} \frac{1}{t_{i}} e^{-\theta / t_{i}}\left(\frac{\sum_{j=1}^{k} j(j-1) a_{j}\left(1-e^{-\theta / t_{i}}\right)^{j-2}}{\sum_{j=1}^{k} j a_{j}\left(1-e^{-\theta / t_{i}}\right)^{j-1}}\right) \\
& +\frac{1}{t^{\prime}} e^{-\theta / t^{\prime}}\left(\frac{\sum_{j=1}^{k} j(j-1) a_{j}\left(1-e^{-\theta / t^{\prime}}\right)^{j-2}}{\sum_{j=1}^{k} j a_{j}\left(1-e^{-\theta / t^{\prime}}\right)^{j-1}}\right) \\
& +(n-m-s) \frac{1}{t^{\prime}} e^{-\theta / t^{\prime}}\left(\frac{\sum_{j=1}^{k} j a_{j}\left(1-e^{-\theta / t^{\prime}}\right)^{j-1}}{\sum_{j=1}^{k} a_{j}\left(1-e^{-\theta / t^{\prime}}\right)^{j}}\right) \\
& +(s-1)\left\{\frac{\frac{1}{t_{m}} e^{-\theta / t_{m}} \sum_{j=1}^{k} j a_{j}\left(1-e^{-\theta / t_{m}}\right)^{j-1}-\frac{1}{t^{\prime}} e^{-\theta / t^{\prime}} \sum_{j=1}^{k} j a_{j}\left(1-e^{-\theta / t^{\prime}}\right)^{j-1}}{\sum_{j=1}^{k} a_{j}\left(1-e^{-\theta / t_{m}}\right)^{j}-\sum_{j=1}^{k} a_{j}\left(1-e^{-\theta / t^{\prime}}\right)^{j}}\right\}=0 .
\end{aligned}
$$

Best unbiased predictor: For $k$-component systems with inverse exponential distributed components, the BUP can be obtained from Eq. (3.9) as

$$
\begin{aligned}
\widehat{T}_{\text {BUP }} & =s\left(\begin{array}{c}
n-m \\
s
\end{array}\right) \theta \sum_{l=0}^{s-1}\left(\begin{array}{c}
s-1 \\
l
\end{array}\right)(-1)^{l}\left(\sum_{j=1}^{k} a_{j}\left(1-e^{-\theta / t_{m}}\right)^{j}\right)^{s-l-1-n+m} \\
& \times \int_{t_{m}}^{\infty} \frac{1}{t^{\prime}} e^{-\theta / t^{\prime}}\left(\sum_{j=1}^{k} j a_{j}\left(1-e^{-\theta / t_{m}}\right)^{j-1}\right)\left(\sum_{j=1}^{k} a_{j}\left(1-e^{-\theta / t_{m}}\right)^{j}\right)^{n-m-s+l} d t^{\prime} .
\end{aligned}
$$

Conditional median predictor: For $k$-component systems with inverse exponential distributed components, since $F_{0}(t)=e^{-t}$, the CMP for $T^{\prime}=T_{s+m: n}(s=1,2, \ldots, n-m)$ can be obtained from Eq. (3.13) by solving the nonlinear equation

$$
\left(\sum_{j=1}^{k} a_{j}\left(1-e^{-\theta / t_{m}}\right)^{j}\right)(1-\operatorname{Med}(B))-\left(\sum_{j=1}^{k} a_{j}\left(1-e^{-\theta / \widehat{T^{\prime}}}\right)^{j}\right)=0 .
$$

Bayesian point predictor: For inverse exponential distributed components, the Bayesian point predictor of the future failure $T^{\prime}=T_{s+m: n}(s=1,2, \ldots, n-m)$ can be obtained from Eq. (3.19) as

$$
\widehat{T}_{\text {Bayes }}=\frac{1}{N} \sum_{i=1}^{N} \sum_{l=0}^{s-1} s\left(\begin{array}{c}
n-m \\
s
\end{array}\right)\left(\begin{array}{c}
s-1 \\
l
\end{array}\right)(-1)^{l} \theta_{i}\left(\sum_{j=1}^{k} a_{j}\left(1-e^{-\theta_{i} / t_{m}}\right)^{j}\right)^{s-l-n+m-1} \xi\left(t_{m} ; \theta_{i}\right),
$$


where

$$
\xi\left(t_{m} ; \theta\right)=\int_{t_{m}}^{\infty} \frac{1}{t^{\prime}} e^{-\theta / t^{\prime}}\left(\sum_{j=1}^{k} j a_{j}\left(1-e^{-\theta / t^{\prime}}\right)^{j-1}\right)\left(\sum_{j=1}^{k} a_{j}\left(1-e^{-\theta / t^{\prime}}\right)^{j}\right)^{n-m-s+l} d t^{\prime} .
$$

\section{Interval Prediction}

In this section, we consider two approaches to obtain the PIs for $T^{\prime}=T_{s+m: n}(1,2, \ldots, n-$ $m$ ) based on the observed Type-II censored sample $\mathbf{t}=\left(t_{1}, t_{2}, \ldots, t_{m}\right)$.

\subsection{Conditional Prediction Interval}

Consider the random variable $Z$ given $T_{m: n}=t_{m}$ as

$$
Z=1-\frac{\sum_{j=1}^{k} a_{j}\left(1-F_{0}^{\theta}\left(T^{\prime}\right)\right)^{j}}{\sum_{j=1}^{k} a_{j}\left(1-F_{0}^{\theta}\left(t_{m}\right)\right)^{j}}
$$

in Section 3, we have shown that the distribution of $Z$ given $\mathbf{T}=\mathbf{t}$ (or simply $\left.T_{m: n}=t_{m}\right)$ follows a $\operatorname{Beta}(s, n-m-s+1)$ distribution. Therefore, we can consider $Z \mid T_{m: n}=t_{m}$ as a pivotal quantity and find a $100(1-\gamma) \%$ PI for $T^{\prime}$ from the relation

$$
\operatorname{Pr}\left(B_{\frac{\gamma}{2}}<Z<B_{1-\frac{\gamma}{2}} \mid T_{m: n}=t_{m}\right)=1-\gamma,
$$

where $B_{\gamma}$ is the $100 \gamma$-th upper percentile of $\operatorname{Beta}(s, n-m-s+1)$ distribution. By solving the inequalities for $T^{\prime}$, an exact $100(1-\gamma) \%$ PI for $T^{\prime}$ is $\left(L_{1}(\mathbf{T}), U_{1}(\mathbf{T})\right)$, where the lower bound $L_{1}(\mathbf{T})$ and upper bound $U_{1}(\mathbf{T})$ are the solutions of

$$
\left(\sum_{j=1}^{k} a_{j}\left(1-F_{0}^{\theta}\left(t_{m}\right)\right)^{j}\right)\left(1-B_{1-\frac{\gamma}{2}}\right)-\left(\sum_{j=1}^{k} a_{j}\left(1-F_{0}^{\theta}\left(T^{\prime}\right)\right)^{j}\right)=0,
$$

and

$$
\left(\sum_{j=1}^{k} a_{j}\left(1-F_{0}^{\theta}\left(t_{m}\right)\right)^{j}\right)\left(1-B_{\frac{\gamma}{2}}\right)-\left(\sum_{j=1}^{k} a_{j}\left(1-F_{0}^{\theta}\left(T^{\prime}\right)\right)^{j}\right)=0,
$$

respectively. However, since $\theta$ is unknown, the prediction limits $L_{1}(\mathbf{T})$ and $U_{1}(\mathbf{T})$ can be approximated by replacing $\theta$ with its corresponding MLE. 
Using the highest conditional density (HCD) method, we can construct another conditional PI for $T^{\prime}$. The conditional distribution of $Z$ given $T_{m: n}=t_{m}$ follows a $\operatorname{Beta}(s, n-m-s+1)$ distribution with PDF

$$
g\left(z \mid t_{m}\right)=\frac{1}{B(s, n-m-s+1)} z^{s-1}(1-z)^{n-m-s}, 0<z<1,
$$

which is a unimodal function of $z$ for $s=1,2, \ldots, n-m$, where $B(a, b)=\int_{0}^{1} x^{a-1}(1-x)^{b-1} d x$ is the beta function. Therefore, a $100(1-\gamma) \%$ HCD PI for $T^{\prime}$ is $\left(L_{2}(\mathbf{T}), U_{2}(\mathbf{T})\right)$, where $L_{2}(\mathbf{T})$ and $U_{2}(\mathbf{T})$ are respectively the solutions of

$$
\left(\sum_{j=1}^{k} a_{j}\left(1-F_{0}^{\theta}\left(t_{m}\right)\right)^{j}\right)\left(1-w_{1}\right)-\left(\sum_{j=1}^{k} a_{j}\left(1-F_{0}^{\theta}\left(T^{\prime}\right)\right)^{j}\right)=0,
$$

and

$$
\left(\sum_{j=1}^{k} a_{j}\left(1-F_{0}^{\theta}\left(t_{m}\right)\right)^{j}\right)\left(1-w_{2}\right)-\left(\sum_{j=1}^{k} a_{j}\left(1-F_{0}^{\theta}\left(T^{\prime}\right)\right)^{j}\right)=0,
$$

with $w_{1}$ and $w_{2}$ satisfy

$$
\int_{w_{1}}^{w_{2}} g\left(z \mid t_{m}\right) d z=1-\gamma
$$

and

$$
g\left(w_{1} \mid t_{m}\right)=g\left(w_{2} \mid t_{m}\right)
$$

Here, Eqs. (4.6) and (4.7) can be simplified as

$$
B_{w_{2}}(s, n-m-s+1)-B_{w_{1}}(s, n-m-s+1)=1-\gamma,
$$

and

$$
\left(\frac{1-w_{2}}{1-w_{1}}\right)^{n-m-s}=\left(\frac{w_{1}}{w_{2}}\right)^{s-1}
$$

where $B_{t}(a, b)=\frac{1}{B(a, b)} \int_{0}^{t} x^{a-1}(1-x)^{b-1} d x$ is the incomplete beta function.

It should be mentioned here that for the case that $s=1$ or $s=n-m$, the function $g\left(z \mid t_{m}\right)$ is not unimodal and the HCD prediction interval cannot be obtained in these cases. 


\subsection{Bayesian Prediction Interval}

A $100(1-\gamma) \%$ Bayesian prediction interval for $T^{\prime}$ can be obtained from the Bayesian predictive density $h^{*}\left(t^{\prime} \mid t_{m} ; \theta\right)$. The $100(1-\gamma) \%$ Bayesian PI for $T^{\prime}$ is given by $\left(L\left(t_{m}\right), U\left(t_{m}\right)\right)$, where $L\left(t_{m}\right)$ and $U\left(t_{m}\right)$ can be obtained by solving the following nonlinear equations simultaneously:

$$
\begin{aligned}
\operatorname{Pr}\left(T^{\prime}>L\left(t_{m}\right) \mid t_{m}\right) & =\int_{L\left(t_{m}\right)}^{\infty} h^{*}\left(t^{\prime} \mid t_{m}\right) d t^{\prime}=1-\frac{\gamma}{2} \\
\operatorname{Pr}\left(T^{\prime}>U\left(t_{m}\right) \mid t_{m}\right) & =\int_{U\left(t_{m}\right)}^{\infty} h^{*}\left(t^{\prime} \mid t_{m}\right) d t^{\prime}=\frac{\gamma}{2} .
\end{aligned}
$$

By using $\widehat{h^{*}}\left(t^{\prime} \mid t_{m}\right)$ defined in Eq. (3.18) to approximate $h^{*}\left(t^{\prime} \mid t_{m}\right)$ and using the MC sample of size $N,\left(\theta_{1}, \theta_{2}, \ldots, \theta_{N}\right)$, from $\pi(\theta \mid \mathbf{t})$, we can compute the lower and upper bounds $L\left(t_{m}\right)$ and $U\left(t_{m}\right)$ from the relations

$$
1-\frac{\gamma}{2}=\frac{1}{N} \sum_{i=1}^{N} \int_{L\left(t_{m}\right)}^{\infty} h\left(t^{\prime} \mid t_{m} ; \theta_{i}\right) d t^{\prime}
$$

and

$$
\frac{\gamma}{2}=\frac{1}{N} \sum_{i=1}^{N} \int_{U\left(t_{m}\right)}^{\infty} h\left(t^{\prime} \mid t_{m} ; \theta_{i}\right) d t^{\prime},
$$

respectively. For the inverse exponential distribution considered in Section 3.4, different PIs can be obtained as described in this section by taking $F_{0}(t)=e^{-\frac{1}{t}}$.

\section{Numerical Illustration and Monte Carlo Simulation Study}

In this section, a numerical example is considered for illustrative purposes and a Monte Carlo simulation study is performed to compare the point and interval prediction methods presented in Sections 3 and 4. It is assumed that the lifetimes of the components are i.i.d. inverse exponential distributed with $\mathrm{CDF}$

$$
F_{X}(t)=e^{-\frac{\theta}{t}}, \quad t>0, \theta>0
$$

which is equivalent to setting $F_{0}(t)=e^{-\frac{1}{t}}$ in Eq. (2.1). 


\subsection{Algorithm to Generate System Lifetimes}

We first discuss the algorithm to generate a random sample of $n$ i.i.d. system lifetimes $T_{1}, T_{2}, \ldots, T_{n}$ for systems with inverse exponential distributed components. For a given system signature $\mathbf{p}=\left(p_{1}, p_{2}, \ldots, p_{k}\right)\left(0<p_{j}<1, \sum_{j=1}^{k} p_{j}=1\right)$, the following algorithm can be used to generate the system lifetimes $T_{1}, T_{2}, \ldots, T_{n}$ with inverse exponential distributed components with specified value of $\theta$ :

Step B1. Generate $u, v_{1}, v_{2}, \ldots, v_{k}$ independently from uniform distribution in $[0,1]$;

Step B2. Set $x_{j}=\theta /\left[-\log \left(v_{j}\right)\right], j=1,2, \ldots, k$;

Step B3. Sort $x_{1}, x_{2}, \ldots, x_{k}$ in ascending order to obtain $x_{1: k}<x_{2: k}<\ldots<x_{k: k}$;

Step B4. Take $t=x_{i: k}$ if $\sum_{j=1}^{i-1} p_{j}<u<\sum_{j=1}^{i} p_{j},(j=1,2, \ldots, k)$, i.e.,

$$
T=\left\{\begin{array}{cc}
x_{1: k} & 0<u<p_{1}, \\
x_{2: k} & p_{1}<u<p_{1}+p_{2}, \\
x_{3: k} & p_{1}+p_{2}<u<p_{1}+p_{2}+p_{3}, \\
\vdots & \vdots \\
x_{k: k} & \sum_{j=1}^{k-1} p_{j}<u<\sum_{j=1}^{k} p_{j} .
\end{array}\right.
$$

Step B5. Repeat Steps 1-4 $n$ times to obtain the system lifetimes $\mathbf{t}=\left(t_{1}, t_{2}, \ldots, t_{n}\right)$.

To obtain a Type-II censored sample based on the simulated system lifetimes $\mathbf{t}=$ $\left(t_{1}, t_{2}, \ldots, t_{n}\right)$, we sort $\left(t_{1}, t_{2}, \ldots, t_{n}\right)$ in ascending order to obtain $t_{1: n}<t_{2: n}<\ldots<t_{n: n}$ and take the first $m$ order statistics $t_{1: n}<t_{2: n}<\ldots<t_{m: n}$ as the Type-II censored sample.

\subsection{Numerical Example}

Using the algorithm provided in Section 5.1, we generate a sample of $n=30$ from a 5component system with system signature $\mathbf{p}=(1 / 5,7 / 10,1 / 10,0,0)$ and the corresponding minimal signature is $\mathbf{a}=(0,0,1,2,-2)$. The component lifetimes are assumed to follow the inverse exponential distribution with exponentiated parameter $\theta=0.5$. The simulated system lifetimes are given in Table 1.

We consider the case when we observe the first 20 observations and the rest are censored, i.e., a Type-II censored sample with $n=30$ and $m=20$ is observed. With this Type-II censored sample, we compute the point and interval prediction for future 
Table 1: Simulated 5-component system lifetimes with system signature $\mathbf{p}=$ $(1 / 5,7 / 10,1 / 10,0,0)$ with inverse exponential distributed components $(\theta=0.5)$.

\begin{tabular}{llllllllll}
\hline 0.136 & 0.146 & 0.190 & 0.201 & 0.204 & 0.224 & 0.237 & 0.249 & 0.250 & 0.258 \\
0.280 & 0.283 & 0.296 & 0.335 & 0.371 & 0.401 & 0.402 & 0.428 & 0.452 & 0.465 \\
0.543 & 0.550 & 0.563 & 0.617 & 0.735 & 0.788 & 0.830 & 0.846 & 1.106 & 1.147 \\
\hline
\end{tabular}

system failures $T^{\prime}=T_{s+20: 30}(s=1,2, \ldots, 10)$ as described in Sections 3 and 4 . Specifically, we compute the MLP, BUP, CMP, Bayesian prediction and we also compute the $95 \%$ PIs for $T^{\prime}=T_{s+20: 30}(s=1,2, \ldots, 10)$ based on the pivotal quantity method, HCD method and Bayesian interval predictor. The results are presented in Table 2.

For the Bayesian prediction, we use the Metropolis-Hastings algorithm to compute the Bayesian point prediction of $T^{\prime}=T_{s+20: 30}(s=1,2, \ldots, 10)$. In the MetropolisHastings algorithm, the MLE $\hat{\theta}$ is considered as the initial value of the chain and the variance of the proposal distribution, $S_{\theta}^{2}$, is obtained by inverting the Fisher information. We sample $N=50000$ values by Metropolis-Hastings algorithm with $S_{\theta}^{2}=0.0027$ and the acceptance rate is about $0.70 \%$. We discard the initial $M=5000$ as burn-in samples and compute the Bayesian prediction $\widehat{T}^{\prime}$ Bayes based on averaging the remaining $N-M=45000$ values. The resulting PIs are also presented in Table 2. For computing the Bayesian PIs, we consider the case that the prior of $\theta$ is almost improper, i.e., $\alpha=\beta=0.0001$.

The histogram of the Metropolis-Hastings sequence for $\theta$ after burn-in is presented in Figure 1. The histogram in Figure 1 can be considered as an approximation of the posterior density of $\theta$ and we can observe that choosing the Gaussian distribution as a proposal distribution is quite appropriate. To evaluate the convergence of MetropolisHastings, graphical diagnostics tools such as the trace plot and autocorrelation function (ACF) plot can be used. The trace plot and ACF plot for the Metropolis-Hastings sequence of values of $\theta$ are also presented in Figure 1. From Figure 1, the trace plot shows the values of $\theta$ are randomly scattered around the average. Furthermore, the ACF plot shows that the sequence has low autocorrelations.

As pointed out in Section 3, the distribution of $Z$ given $T_{m: n}=t_{m}$ is a unimodal function of $z$, for $s=1,2, \ldots, n-m$. Therefore, the HCD prediction method is applicable here for all $T^{\prime}=T_{s+25: 30}(s=2,3,4,5,6,7,8,9)$ except for $s=1$ and $s=10$. From Table 2, we observe that the BUP and Bayes point predictor are close to the realized censored 
lifetimes. We also observe that the PI's obtained using the Bayesian method are shorter than the PI's obtained from other techniques, and the prediction intervals considered here contain the realized censored lifetimes.

Table 2: Point predictors and 95\% prediction intervals for $T^{\prime}$ based on the data set in Table 1.

\begin{tabular}{|c|c|c|c|c|c|c|c|c|}
\hline \multirow[b]{2}{*}{$s$} & \multirow{2}{*}{$\begin{array}{l}\text { Exact } \\
\text { value }\end{array}$} & \multicolumn{4}{|c|}{ Point predictors } & \multicolumn{3}{|c|}{ Prediction intervals } \\
\hline & & MLP & BUP & CMP & Bayes & Pivotal & HCD & Bayesian \\
\hline 1 & 0.543 & 0.465 & 0.494 & 0.485 & 0.494 & $(0.466,0.581)$ & - & $(0.466,0.574)$ \\
\hline 2 & 0.550 & 0.495 & 0.527 & 0.516 & 0.527 & $(0.473,0.652)$ & $(0.468,0.625)$ & $(0.473,0.643)$ \\
\hline 3 & 0.563 & 0.528 & 0.564 & 0.552 & 0.565 & $(0.487,0.728)$ & $(0.480,0.703)$ & $(0.485,0.717)$ \\
\hline 4 & 0.617 & 0.566 & 0.608 & 0.594 & 0.609 & $(0.505,0.816)$ & $(0.500,0.796)$ & $(0.502,0.801)$ \\
\hline 5 & 0.735 & 0.611 & 0.661 & 0.644 & 0.663 & $(0.530,0.924)$ & $(0.527,0.915)$ & $(0.525,0.905)$ \\
\hline 6 & 0.788 & 0.665 & 0.728 & 0.706 & 0.730 & $(0.560,1.064)$ & $(0.563,1.077)$ & $(0.552,1.038)$ \\
\hline 7 & 0.830 & 0.733 & 0.817 & 0.787 & 0.820 & $(0.599,1.263)$ & $(0.609,1.328)$ & $(0.589,1.232)$ \\
\hline 8 & 0.846 & 0.825 & 0.948 & 0.902 & 0.950 & $(0.652,1.585)$ & $(0.673,1.810)$ & $(0.637,1.538)$ \\
\hline 9 & 1.106 & 0.959 & 1.178 & 1.090 & 1.182 & $(0.727,2.251)$ & $(0.767,3.455)$ & $(0.709,2.182)$ \\
\hline 10 & 1.147 & 1.201 & 1.841 & 1.525 & 1.848 & $(0.861,4.943)$ & - & $(0.836,4.778)$ \\
\hline
\end{tabular}
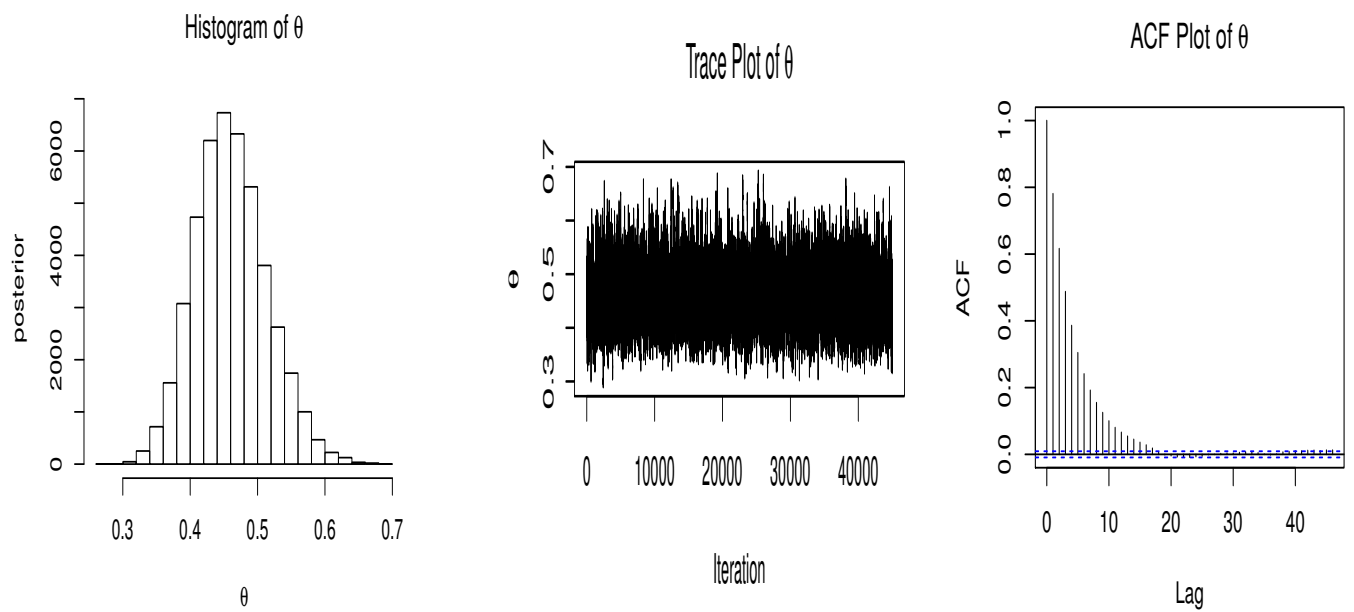

Figure 1: Plots of Metropolis-Hastings Markov chains for $\theta$. 


\subsection{Monte Carlo Simulation Study}

In order to compare the performance of different point and interval prediction methods presented in this paper, we perform a Monte Carlo simulation study for systems with inverse exponential distributed components. In this simulation, we consider 7 different 5-component systems. The minimal path sets, system signatures, and minimal signatures of the 5-component systems considered in the simulation study are presented in Table 3 (see, for example, Navarro and Rubio, 2010).

For different choices of sample size $n$ and effective sample size $m$, we generated 1000 sets of censored system lifetimes $T_{1: n}<T_{2: n}<\ldots<T_{m: n}$ with inverse exponential distributed components with parameter $\theta=0.5$ using the algorithm in Section 5.1. We then obtained the point predictors MLP, BUP, CMP for the $s$-th future system failure time $T^{\prime}=T_{s+m: n}(s=1,2, \ldots, n-m)$. We also obtained Bayesian point prediction under two different priors:

$$
\begin{aligned}
& \text { Prior I: } \quad \alpha=2, \beta=4 ; \\
& \text { Prior II: } \quad \alpha=\beta=0.0001 .
\end{aligned}
$$

The performances of the different point predictors of $T^{\prime}$ are then compared in terms of the prediction biases and mean squares prediction errors (MSPEs) which are computed by

$$
\text { Bias }=\frac{1}{1000} \sum_{i=1}^{1000}\left(\hat{T}^{\prime}{ }_{i}-T^{\prime}\right) \text { and MSPE }=\frac{1}{1000} \sum_{i=1}^{1000}\left(\hat{T}^{\prime}{ }_{i}-T^{\prime}\right)^{2}
$$

respectively, where $\hat{T}^{\prime}{ }_{i}$ is the point prediction of $T^{\prime}$ obtained in the $i$-th simulation. In Table 4, the estimated biases and MSPEs for different point predictors based on 1000 replications for sample sizes $(n=10, m=7)$ and $(n=25, m=20)$. The computations are performed in R (R Core Team, 2019) with the MHadaptive package (Chivers, 2012).

For interval prediction, we compute the 95\% PIs for $T^{\prime}=T_{s+m: n}(1,2, \ldots, n-m)$ based on the pivotal quantity method, the HCD Method and the Bayesian interval predictor based on the Priors I and II. These prediction intervals are compared in terms of their simulated average widths and simulated coverage probabilities based on 1000 replications. The results for sample sizes $(n=10, m=7)$ and $(n=25, m=20)$ are reported in Tables 5 and 6, respectively.

For point prediction, from Table 4, we observe that BUP performs better that the MLP and CMP in terms of biases and MSPEs. The MSPEs of CMP and the MSPEs of BUP 
are close to each other. For fixed value of $m$ and $n$ and the system structure, the MSPEs of all the point predictors are increasing with $s$ as expected. Comparing the Bayesian point predictions based on different priors, the Bayesian point predictors based on informative priors (i.e., Prior I) perform better than the Bayesian point predictors based on the non-informative prior (i.e., Prior II), in terms of biases and MSPEs. However, the biases and MSPEs of the two Bayesian point predictors are similar. We also observe that the MLP does not perform well because it provides the largest biases and MSPEs among all the point predictors considered here.

For prediction intervals, we observe from Tables 5 and 6 that the simulated coverage probabilities are close to the nominal level $95 \%$ in most cases. It can be seen that Bayesian PIs are wider than the PIs obtained by the pivotal quantity method and the HCD method. When the informative Prior I is used, the average length of the PIs become smaller. For fixed values of $n$ and $m$ and the system structure, the average widths of different prediction intervals increase as $s$ increases. Among the methods for constructing prediction intervals considered here, the pivotal quantity method can provide prediction intervals for all the censored system failures and it gives the best performance in terms of coverage probabilities and average widths.

Table 3: Minimal path sets, system signatures, and minimal signatures of the 5component systems considered in the simulation study.

\begin{tabular}{cccc}
\hline System No. & Minimal Path Sets & Signature $\mathbf{p}$ & Minimal Signature a \\
\hline 1 & $\{1,2,3,4,5\}$ & $(1,0,0,0,0)$ & $(0,0,0,0,1)$ \\
2 & $\{1,2,3,4\},\{1,2,3,5\},\{1,2,4,5\}$ & $\left(\frac{2}{5}, \frac{3}{5}, 0,0,0\right)$ & $(0,0,0,3,-2)$ \\
3 & $\{1,2,3,4\},\{1,2,3,5\}$ & $\left(\frac{3}{5}, \frac{2}{5}, 0,0,0\right)$ & $(0,0,0,2,-1)$ \\
4 & $\{1,2,3\},\{1,2,4\},\{1,2,5\},\{1,3,4\},\{1,3,5\}$ & $\left(\frac{1}{5}, \frac{3}{10}, \frac{1}{2}, 0,0\right)$ & $(0,0,5,-6,2)$ \\
5 & $\{1,2,3\},\{1,2,4\},\{1,2,5\}$ & $\left(\frac{2}{5}, \frac{3}{10}, \frac{3}{10}, 0,0\right)$ & $(0,0,3,-3,1)$ \\
6 & $\{1,2\},\{1,3,4,5\},\{2,3,4,5\}$ & $\left(0, \frac{7}{10}, \frac{1}{5}, \frac{1}{10}, 0\right)$ & $(0,1,0,2,-2)$ \\
7 & $\{1,2,3\},\{1,2,4,5\}$ & $\left(\frac{2}{5}, \frac{1}{2}, \frac{1}{10}, 0,0\right)$ & $(0,0,1,1,-1)$ \\
\hline
\end{tabular}


Table 4: Biases and MSPEs (in parentheses) of point predictions for $T^{\prime}=T_{s+m: n}(s=$ $1,2, \ldots, n-m)$.

\begin{tabular}{|c|c|c|c|c|c|c|c|c|}
\hline \multirow[b]{2}{*}{$\mathrm{n}$} & \multirow[b]{2}{*}{$\mathrm{m}$} & \multirow[b]{2}{*}{ no. } & \multirow[b]{2}{*}{$s$} & \multicolumn{3}{|c|}{ Classic point predictions } & \multicolumn{2}{|c|}{ Bayesian point predictions } \\
\hline & & & & MLP & BUP & CMP & Prior I & Prior II \\
\hline \multirow[t]{21}{*}{10} & \multirow[t]{21}{*}{7} & \multirow[t]{3}{*}{1} & 1 & $-0.0526(0.0058)$ & $0.0010(0.0032)$ & $-0.0159(0.0034)$ & $0.0019(0.0032)$ & $0.0021(0.0032)$ \\
\hline & & & 2 & $-0.0843(0.0194)$ & $-0.0069(0.0125)$ & $-0.0343(0.0135)$ & $-0.0052(0.0125)$ & $-0.0049(0.0126)$ \\
\hline & & & 3 & $-0.1616(0.0969)$ & $0.0147(0.0720)$ & $-0.0591(0.0746)$ & $0.0178(0.0720)$ & $0.0189(0.0721)$ \\
\hline & & \multirow[t]{3}{*}{2} & 1 & $-0.0910(0.0177)$ & $0.0010(0.0095)$ & $-0.0291(0.0102)$ & $0.0030(0.0095)$ & $0.0032(0.0095)$ \\
\hline & & & 2 & $-0.1280(0.0482)$ & $0.0130(0.0333)$ & $-0.0391(0.0340)$ & $0.0160(0.0333)$ & $0.0170(0.0335)$ \\
\hline & & & 3 & $-0.3430(0.4291)$ & $0.0071(0.3198)$ & $-0.1480(0.3368)$ & $0.0140(0.3189)$ & $0.0160(0.3206)$ \\
\hline & & \multirow[t]{3}{*}{3} & 1 & $-0.0834(0.0152)$ & $-0.0002(0.0084)$ & $-0.0274(0.0090)$ & $0.0008(0.0084)$ & $0.0013(0.0084)$ \\
\hline & & & 2 & $0.1267(0.0521)$ & $0.0002(0.0367)$ & $-0.0464(0.0385)$ & $0.0024(0.0366)$ & $0.0034(0.0368)$ \\
\hline & & & 3 & $-0.3092(0.3806)$ & $0.0096(0.2898)$ & $-0.1321(0.3040)$ & $0.0141(0.2888)$ & $0.0160(0.2901)$ \\
\hline & & \multirow[t]{3}{*}{4} & 1 & $-0.1700(0.0645)$ & $0.0008(0.0362)$ & $-0.0578(0.0390)$ & $0.0034(0.0361)$ & $0.0049(0.0363)$ \\
\hline & & & 2 & $-0.2713(0.2316)$ & $0.0223(0.1652)$ & $-0.0921(0.1693)$ & $0.0281(0.1643)$ & $0.0321(0.1662)$ \\
\hline & & & 3 & $-0.8372(4.1520)$ & $0.0284(3.4825)$ & $-0.3957(3.6114)$ & $0.0392(3.4778)$ & $0.0502(3.4873)$ \\
\hline & & \multirow[t]{3}{*}{5} & 1 & $-0.1464(0.0498)$ & $-0.0009(0.0282)$ & $-0.0512(0.0306)$ & $0.0002(0.0281)$ & $0.0011(0.0283)$ \\
\hline & & & 2 & $-0.2385(0.1748)$ & $0.0078(0.1209)$ & $-0.0883(0.1265)$ & $0.0099(0.1204)$ & $0.0123(0.1211)$ \\
\hline & & & 3 & $-0.7161(3.2973)$ & $0.0106(2.7838)$ & $-0.3454(2.8949)$ & $0.0138(2.7782)$ & $0.0221(2.7856)$ \\
\hline & & \multirow[t]{3}{*}{6} & 1 & $-0.1730(0.0698)$ & $0.0150(0.0393)$ & $-0.0550(0.0418)$ & $0.0160(0.0393)$ & $0.0170(0.0394)$ \\
\hline & & & 2 & $-0.3640(0.4488)$ & $0.0030(0.3078)$ & $-0.1590(0.3345)$ & $0.0050(0.3077)$ & $0.0060(0.3080)$ \\
\hline & & & 3 & $-1.1980(3.5293)$ & $0.3010(2.4380)$ & $-0.5991(2.4895)$ & $0.3054(2.4352)$ & $0.3132(2.4456)$ \\
\hline & & \multirow[t]{3}{*}{7} & 1 & $-0.1110(0.0282)$ & $-0.0020(0.0158)$ & $-0.0390(0.0172)$ & $-0.0010(0.0158)$ & $0.0000(0.0159)$ \\
\hline & & & 2 & $-0.1700(0.0901)$ & $0.0080(0.0628)$ & $-0.0610(0.0654)$ & $0.0111(0.0626)$ & $0.0133(0.0630)$ \\
\hline & & & 3 & $-0.5001(1.3592)$ & $0.0100(1.0973)$ & $-0.2380(1.1561)$ & $0.0172(1.0981)$ & $0.0200(1.0993)$ \\
\hline \multirow[t]{35}{*}{25} & 20 & 1 & 1 & $-0.0337(0.0023)$ & $-0.0012(0.0011)$ & $-0.0115(0.0013)$ & $-0.0011(0.0011)$ & $-0.0010(0.0011)$ \\
\hline & & & 2 & $-0.0386(0.0047)$ & $0.0010(0.0032)$ & $-0.0124(0.0034)$ & $0.0013(0.0032)$ & $0.0014(0.0032)$ \\
\hline & & & 3 & $-0.0495(0.0092)$ & $0.0032(0.0069)$ & $-0.0153(0.0071)$ & $0.0038(0.0069)$ & $0.0038(0.0070)$ \\
\hline & & & 4 & $-0.0852(0.0294)$ & $-0.0023(0.0223)$ & $-0.0331(0.0233)$ & $-0.0016(0.0223)$ & $-0.0014(0.0224)$ \\
\hline & & & 5 & $-0.2152(0.1939)$ & $-0.0138(0.1492)$ & $-0.1005(0.1583)$ & $-0.0125(0.1490)$ & $-0.0122(0.1492)$ \\
\hline & & 2 & 1 & $-0.0580(0.0075)$ & $-0.0010(0.0041)$ & $-0.0200(0.0044)$ & $-0.0010(0.0041)$ & $-0.0010(0.0041)$ \\
\hline & & & 2 & $-0.0680(0.0134)$ & $0.0030(0.0089)$ & $-0.0211(0.0093)$ & $0.0040(0.0089)$ & $0.0040(0.0089)$ \\
\hline & & & 3 & $-0.1030(0.0363)$ & $-0.0040(0.0259)$ & $-0.0391(0.0273)$ & $-0.0030(0.0259)$ & $-0.0020(0.0260)$ \\
\hline & & & 4 & $-0.1560(0.0977)$ & $0.0080(0.0742)$ & $-0.0550(0.0767)$ & $0.0091(0.0742)$ & $0.0100(0.0744)$ \\
\hline & & & 5 & $-0.4210(0.7360)$ & $0.0081(0.5565)$ & $-0.1870(0.5916)$ & $0.0110(0.5567)$ & $0.0111(0.5569)$ \\
\hline & & 3 & 1 & $-0.0518(0.0062)$ & $-0.0008(0.0035)$ & $-0.0173(0.0038)$ & $-0.0006(0.0035)$ & $-0.0006(0.0035)$ \\
\hline & & & 2 & $-0.0578(0.0100)$ & $0.0065(0.0068)$ & $-0.0156(0.0069)$ & $0.0069(0.0068)$ & $0.0070(0.0068)$ \\
\hline & & & 3 & $-0.0864(0.0250)$ & $0.0018(0.0177)$ & $-0.0299(0.0185)$ & $0.0025(0.0177)$ & $0.0028(0.0178)$ \\
\hline & & & 4 & $-0.1408(0.0820)$ & $0.0080(0.0629)$ & $-0.0491(0.0649)$ & $0.0090(0.0629)$ & $0.0094(0.0631)$ \\
\hline & & & 5 & $-0.3857(0.9031)$ & $-0.0012(0.7516)$ & $-0.1760(0.7831)$ & $0.0010(0.7512)$ & $0.0012(0.7513)$ \\
\hline & & 4 & 1 & $-0.1072(0.0234)$ & $0.0032(0.0119)$ & $-0.0334(0.0129)$ & $0.0038(0.0119)$ & $0.0039(0.0119)$ \\
\hline & & & 2 & $-0.1446(0.0751)$ & $0.0023(0.0540)$ & $-0.0499(0.0565)$ & $0.0035(0.0540)$ & $0.0038(0.0543)$ \\
\hline & & & 3 & $-0.2059(0.1750)$ & $0.0091(0.1336)$ & $-0.0712(0.1379)$ & $0.0112(0.1336)$ & $0.0119(0.1338)$ \\
\hline & & & 4 & $-0.3751(0.4784)$ & $0.0074(0.3374)$ & $-0.1471(0.3580)$ & $0.0106(0.3373)$ & $0.0117(0.3378)$ \\
\hline & & & 5 & $-1.1285(6.1137)$ & $0.0004(4.8550)$ & $-0.5616(5.1527)$ & $0.0073(4.8499)$ & $0.0085(4.8564)$ \\
\hline & & 5 & 1 & $-0.0865(0.0160)$ & $0.0058(0.0085)$ & $-0.0248(0.0091)$ & $0.0061(0.0085)$ & $0.0062(0.0085)$ \\
\hline & & & 2 & $-0.1247(0.0458)$ & $-0.0015(0.0304)$ & $-0.0453(0.0323)$ & $-0.0009(0.0304)$ & $-0.0007(0.0304)$ \\
\hline & & & 3 & $-0.1794(0.1103)$ & $0.0016(0.0779)$ & $-0.0660(0.0821)$ & $0.0025(0.0779)$ & $0.0029(0.0780)$ \\
\hline & & & 4 & $-0.3046(0.3378)$ & $0.0133(0.2473)$ & $-0.1150(0.2587)$ & $0.0150(0.2472)$ & $0.0158(0.2474)$ \\
\hline & & & 5 & $-1.000(4.6531)$ & $-0.0465(3.6695)$ & $-0.5211(3.9258)$ & $-0.0432(3.6674)$ & $-0.0425(3.6706)$ \\
\hline & & 6 & 1 & $-0.1327(0.0407)$ & $-0.0084(0.0231)$ & $-0.0513(0.0257)$ & $-0.0081(0.0231)$ & $-0.0080(0.0231)$ \\
\hline & & & 2 & $-0.1778(0.0982)$ & $0.0011(0.0666)$ & $-0.0668(0.0708)$ & $0.0025(0.0666)$ & $0.0029(0.0668)$ \\
\hline & & & 3 & $-0.2931(0.3045)$ & $0.0000(0.2131)$ & $-0.1190(0.2286)$ & $0.0013(0.2132)$ & $0.0016(0.2135)$ \\
\hline & & & 4 & $-0.5510(1.0862)$ & $0.0470(0.7758)$ & $-0.2230(0.8239)$ & $0.0480(0.7753)$ & $0.0481(0.7762)$ \\
\hline & & & 5 & $-1.4480(3.4496)$ & $0.8840(2.2851)$ & $-0.7750(2.7290)$ & $0.8830(2.2794)$ & $0.8891(2.2944)$ \\
\hline & & 7 & 1 & $-0.0710(0.0107)$ & $-0.0020(0.0057)$ & $-0.0251(0.0063)$ & $-0.0020(0.0057)$ & $-0.0020(0.0057)$ \\
\hline & & & 2 & $-0.0881(0.0236)$ & $0.0020(0.0161)$ & $-0.0301(0.0169)$ & $0.0020(0.0161)$ & $0.0021(0.0162)$ \\
\hline & & & 3 & $-0.1260(0.0564)$ & $0.0051(0.0409)$ & $-0.0442(0.0425)$ & $0.0060(0.0408)$ & $0.0060(0.0409)$ \\
\hline & & & 4 & $-0.2372(0.1983)$ & $-0.0080(0.1431)$ & $-0.1000(0.1523)$ & $-0.0071(0.1431)$ & $-0.0071(0.1432)$ \\
\hline & & & 5 & $-0.6310(1.5894)$ & $0.0342(1.1836)$ & $-0.2960(1.2713)$ & $0.0370(1.1841)$ & $0.0380(1.1844)$ \\
\hline
\end{tabular}


Table 5: Simulated average widths (AW) and coverage probabilities (CP) of 95\% PIs of $T^{\prime}$.

\begin{tabular}{|c|c|c|c|c|c|c|c|c|}
\hline \multirow[b]{2}{*}{$n$} & \multirow[b]{2}{*}{$m$} & \multirow[b]{2}{*}{ no. } & \multirow[b]{2}{*}{$s$} & & \multirow[b]{2}{*}{ Pivotal } & \multirow[b]{2}{*}{$\mathrm{HCD}$} & \multicolumn{2}{|l|}{ Prediction Intervals } \\
\hline & & & & & & & Prior I & Prior II \\
\hline \multirow[t]{42}{*}{10} & 7 & 1 & 1 & $\mathrm{AW}$ & 0.199 & - & 0.208 & 0.209 \\
\hline & & & & $\mathrm{CP}$ & 0.944 & - & 0.937 & 0.937 \\
\hline & & & 2 & $\mathrm{AW}$ & 0.401 & 0.401 & 0.420 & 0.422 \\
\hline & & & & $\mathrm{CP}$ & 0.959 & 0.959 & 0.957 & 0.955 \\
\hline & & & 3 & $\mathrm{AW}$ & 0.988 & - & 1.031 & 1.033 \\
\hline & & & & $\mathrm{CP}$ & 0.949 & - & 0.947 & 0.948 \\
\hline & & 2 & 1 & AW & 0.346 & - & 0.360 & 0.363 \\
\hline & & & & $\mathrm{CP}$ & 0.936 & - & 0.939 & 0.939 \\
\hline & & & 2 & $\mathrm{AW}$ & 0.727 & 0.727 & 0.757 & 0.761 \\
\hline & & & & $\mathrm{CP}$ & 0.958 & 0.958 & 0.955 & 0.954 \\
\hline & & & 3 & $\mathrm{AW}$ & 1.992 & - & 2.070 & 2.081 \\
\hline & & & & $\mathrm{CP}$ & 0.964 & - & 0.959 & 0.958 \\
\hline & & 3 & 1 & AW & 0.313 & - & 0.327 & 0.329 \\
\hline & & & & $\mathrm{CP}$ & 0.956 & - & 0.956 & 0.955 \\
\hline & & & 2 & $\mathrm{AW}$ & 0.649 & 0.649 & 0.673 & 0.677 \\
\hline & & & & $\mathrm{CP}$ & 0.944 & 0.944 & 0.943 & 0.941 \\
\hline & & & 3 & $\mathrm{AW}$ & 1.783 & - & 1.833 & 1.842 \\
\hline & & & & $\mathrm{CP}$ & 0.939 & - & 0.937 & 0.935 \\
\hline & & 4 & 1 & $\mathrm{AW}$ & 0.675 & - & 0.705 & 0.713 \\
\hline & & & & $\mathrm{CP}$ & 0.938 & - & 0.943 & 0.944 \\
\hline & & & 2 & $\mathrm{AW}$ & 1.502 & 1.502 & 1.554 & 1.570 \\
\hline & & & & $\mathrm{CP}$ & 0.960 & 0.960 & 0.960 & 0.958 \\
\hline & & & 3 & AW & 4.759 & - & 4.897 & 4.935 \\
\hline & & & & $\mathrm{CP}$ & 0.941 & - & 0.945 & 0.944 \\
\hline & & 5 & 1 & AW & 0.562 & - & 0.583 & 0.587 \\
\hline & & & & $\mathrm{CP}$ & 0.954 & - & 0.959 & 0.955 \\
\hline & & & 2 & AW & 1.271 & 1.271 & 1.322 & 1.332 \\
\hline & & & & $\mathrm{CP}$ & 0.940 & 0.940 & 0.939 & 0.941 \\
\hline & & & 3 & $\mathrm{AW}$ & 4.037 & - & 4.152 & 4.179 \\
\hline & & & & $\mathrm{CP}$ & 0.949 & - & 0.957 & 0.956 \\
\hline & & 6 & 1 & AW & 0.762 & - & 0.777 & 0.779 \\
\hline & & & & $\mathrm{CP}$ & 0.947 & - & 0.947 & 0.948 \\
\hline & & & 2 & AW & 1.939 & 1.939 & 1.963 & 1.969 \\
\hline & & & & $\mathrm{CP}$ & 0.947 & 0.947 & 0.946 & 0.944 \\
\hline & & & 3 & AW & 8.547 & - & 8.639 & 8.659 \\
\hline & & & & $\mathrm{CP}$ & 0.951 & - & 0.952 & 0.952 \\
\hline & & 7 & 1 & AW & 0.420 & - & 0.437 & 0.441 \\
\hline & & & & $\mathrm{CP}$ & 0.953 & - & 0.956 & 0.959 \\
\hline & & & 2 & AW & 0.926 & 0.926 & 0.964 & 0.972 \\
\hline & & & & $\mathrm{CP}$ & 0.931 & 0.930 & 0.933 & 0.933 \\
\hline & & & 3 & AW & 2.890 & - & 2.976 & 2.992 \\
\hline & & & & $\mathrm{CP}$ & 0.945 & - & 0.942 & 0.939 \\
\hline \multirow[t]{20}{*}{25} & 20 & 1 & 1 & AW & 0.121 & - & 0.122 & 0.123 \\
\hline & & & & $\mathrm{CP}$ & 0.952 & - & 0.953 & 0.952 \\
\hline & & & 2 & AW & 0.206 & 0.183 & 0.208 & 0.209 \\
\hline & & & & $\mathrm{CP}$ & 0.949 & 0.945 & 0.944 & 0.943 \\
\hline & & & 3 & AW & 0.319 & 0.319 & 0.324 & 0.324 \\
\hline & & & & $\mathrm{CP}$ & 0.953 & 0.953 & 0.952 & 0.952 \\
\hline & & & 4 & AW & 0.525 & 0.704 & 0.531 & 0.532 \\
\hline & & & & $\mathrm{CP}$ & 0.950 & 0.955 & 0.950 & 0.950 \\
\hline & & & 5 & AW & 1.216 & - & 1.226 & 1.227 \\
\hline & & & & $\mathrm{CP}$ & 0.953 & - & 0.949 & 0.948 \\
\hline & & 2 & 1 & AW & 0.212 & - & 0.214 & 0.215 \\
\hline & & & & $\mathrm{CP}$ & 0.944 & - & 0.943 & 0.943 \\
\hline & & & 2 & AW & 0.371 & 0.327 & 0.377 & 0.378 \\
\hline & & & & $\mathrm{CP}$ & 0.956 & 0.956 & 0.956 & 0.957 \\
\hline & & & 3 & AW & 0.587 & 0.587 & 0.596 & 0.597 \\
\hline & & & & $\mathrm{CP}$ & 0.963 & 0.963 & 0.960 & 0.961 \\
\hline & & & 4 & AW & 1.006 & 1.383 & 1.021 & 1.022 \\
\hline & & & & $\mathrm{CP}$ & 0.946 & 0.948 & 0.942 & 0.943 \\
\hline & & & 5 & AW & 2.535 & - & 2.566 & 2.570 \\
\hline & & & & $\mathrm{CP}$ & 0.936 & - & 0.940 & 0.942 \\
\hline
\end{tabular}


Table 6: Continued.

\begin{tabular}{|c|c|c|c|c|c|c|c|c|}
\hline \multirow[b]{2}{*}{$n$} & \multirow[b]{2}{*}{$m$} & \multirow[b]{2}{*}{ no. } & \multirow[b]{2}{*}{$s$} & & \multirow[b]{2}{*}{ Pivotal } & \multicolumn{3}{|c|}{ Prediction Intervals } \\
\hline & & & & & & HCD & Prior I & Prior II \\
\hline \multirow[t]{50}{*}{25} & 20 & 3 & 1 & AW & 0.191 & - & 0.193 & 0.193 \\
\hline & & & & $\mathrm{CP}$ & 0.943 & - & 0.945 & 0.945 \\
\hline & & & 2 & AW & 0.334 & 0.294 & 0.338 & 0.339 \\
\hline & & & & $\mathrm{CP}$ & 0.950 & 0.956 & 0.954 & 0.954 \\
\hline & & & 3 & AW & 0.528 & 0.528 & 0.537 & 0.538 \\
\hline & & & & $\mathrm{CP}$ & 0.955 & 0.955 & 0.956 & 0.955 \\
\hline & & & 4 & AW & 0.904 & 1.243 & 0.918 & 0.919 \\
\hline & & & & $\mathrm{CP}$ & 0.964 & 0.959 & 0.965 & 0.964 \\
\hline & & & 5 & AW & 2.284 & - & 2.306 & 2.306 \\
\hline & & & & $\mathrm{CP}$ & 0.957 & - & 0.950 & 0.949 \\
\hline & & 4 & 1 & AW & 0.420 & - & 0.426 & 0.427 \\
\hline & & & & $\mathrm{CP}$ & 0.946 & - & 0.946 & 0.948 \\
\hline & & & 2 & AW & 0.755 & 0.660 & 0.765 & 0.766 \\
\hline & & & & $\mathrm{CP}$ & 0.959 & 0.964 & 0.960 & 0.958 \\
\hline & & & 3 & $\mathrm{AW}$ & 1.257 & 1.257 & 1.277 & 1.280 \\
\hline & & & & $\mathrm{CP}$ & 0.954 & 0.954 & 0.953 & 0.953 \\
\hline & & & 4 & AW & 2.255 & 3.233 & 2.274 & 2.276 \\
\hline & & & & $\mathrm{CP}$ & 0.943 & 0.941 & 0.943 & 0.942 \\
\hline & & & 5 & $\mathrm{AW}$ & 6.653 & - & 6.730 & 6.737 \\
\hline & & & & $\mathrm{CP}$ & 0.958 & - & 0.955 & 0.955 \\
\hline & & 5 & 1 & AW & 0.352 & - & 0.357 & 0.358 \\
\hline & & & & $\mathrm{CP}$ & 0.945 & - & 0.946 & 0.947 \\
\hline & & & 2 & $\mathrm{AW}$ & 0.636 & 0.555 & 0.645 & 0.646 \\
\hline & & & & $\mathrm{CP}$ & 0.957 & 0.947 & 0.951 & 0.952 \\
\hline & & & 3 & AW & 1.046 & 1.046 & 1.061 & 1.062 \\
\hline & & & & CP & 0.961 & 0.961 & 0.963 & 0.963 \\
\hline & & & 4 & AW & 1.911 & 2.741 & 1.939 & 1.940 \\
\hline & & & & CP & 0.954 & 0.953 & 0.954 & 0.953 \\
\hline & & & 5 & AW & 5.594 & - & 5.657 & 5.662 \\
\hline & & & & $\mathrm{CP}$ & 0.963 & - & 0.963 & 0.964 \\
\hline & & 6 & 1 & AW & 0.483 & - & 0.484 & 0.484 \\
\hline & & & & $\mathrm{CP}$ & 0.954 & - & 0.955 & 0.955 \\
\hline & & & 2 & AW & 0.923 & 0.791 & 0.925 & 0.926 \\
\hline & & & & $\mathrm{CP}$ & 0.958 & 0.950 & 0.959 & 0.959 \\
\hline & & & 3 & AW & 1.643 & 1.643 & 1.648 & 1.649 \\
\hline & & & & CP & 0.945 & 0.945 & 0.943 & 0.943 \\
\hline & & & 4 & AW & 3.472 & 5.458 & 3.482 & 3.487 \\
\hline & & & & $\mathrm{CP}$ & 0.942 & 0.942 & 0.941 & 0.941 \\
\hline & & & 5 & AW & 8.851 & - & 8.885 & 8.889 \\
\hline & & & & CP & 0.951 & - & 0.954 & 0.954 \\
\hline & & 7 & 1 & AW & 0.261 & - & 0.264 & 0.266 \\
\hline & & & & $\mathrm{CP}$ & 0.954 & - & 0.952 & 0.952 \\
\hline & & & 2 & AW & 0.464 & 0.406 & 0.469 & 0.470 \\
\hline & & & & $\mathrm{CP}$ & 0.944 & 0.950 & 0.948 & 0.949 \\
\hline & & & 3 & AW & 0.759 & 0.759 & 0.769 & 0.770 \\
\hline & & & & $\mathrm{CP}$ & 0.949 & 0.949 & 0.950 & 0.950 \\
\hline & & & 4 & AW & 1.368 & 1.951 & 1.383 & 1.384 \\
\hline & & & & $\mathrm{CP}$ & 0.959 & 0.951 & 0.960 & 0.960 \\
\hline & & & 5 & AW & 3.910 & - & 3.941 & 3.942 \\
\hline & & & & CP & 0.942 & - & 0.940 & 0.940 \\
\hline
\end{tabular}

\section{Concluding Remarks}

In this paper, we discuss the prediction problem based on Type-II censored system lifetime data when the system structure is known and the component lifetime follows the proportional reversed hazard model. Different point predictors for censored system failures, including the maximum likelihood predictor, the best unbiased predictor, 
the conditional median predictor, and the Bayesian point predictor, are developed. We also developed the prediction intervals for the censored system failures using pivotal quantity method, highest conditional density method and Bayesian method. A numerical example is presented to illustrate the prediction methods by considering the component lifetimes follow the inverse exponential distribution. Monte Carlo simulation study is used to evaluate the performance of the point and interval prediction methods considered in this paper. Based on the simulation results, we would recommend using the best unbiased predictor for point prediction unless reliable prior information on the unknown parameter is available. For interval prediction, we suggest using the pivotal quantity method to construct prediction intervals for the censored system failures.

\section{Acknowledgements}

The authors thank the guest editor, Professor Syed N. U. A. Kirmani, for giving them the opportunity to contribute to this Behboodian Memorial issue and thank the anonymous reviewers for reviewing the manuscripts. H.K.T. $\mathrm{Ng}^{\prime}$ 's research is supported by a grant from the Simons Foundation (\#709773 to Tony Ng).

\section{References}

Asgharzadeh, A. Fallah, A., Raqab, M. Z. and Valiollahi, R. (2018), Statistical inference based on Lindley record data. Statistical Papers, 59, 759-779.

Asgharzadeh, A., Valiollahi, R. and Kundu, D. (2015), Prediction for future failures in Weibull distribution under hybrid censoring. Journal of Statistical Computation and Simulation, 85, 824-838.

Balakrishnan, N., Ng, H. K. T. and Navarro, J. (2011a), Linear inference for Type-II censored lifetime data of reliability systems with known signatures. IEEE Transactions on Reliability, 60, 426-440.

Balakrishnan, N., Ng, H. K. T., and Navarro, J. (2011b), Exact nonparametric inference for component lifetime distribution based on lifetime data from systems with known signatures. Journal of Nonparametric Statistics, 23, 741-752.

Bhattacharya and Samaniego (2010), Estimating component characteristics from system failure time data. Naval Research Logistics, 57, 380-389. 
Basak, I., Basak, P., and Balakrishnan, N. (2006), On some predictors of times to failures of censored items in progressively censored sample. Computational Statistics and Data Analysis, 50, 1313-1337.

Basak, I., and N. Balakrishnan, N. (2017), Prediction of censored exponential lifetimes in a simple step-stress model under progressive Type II censoring. Computational Statistics, 32, 1665-1687.

Chahkandi, M., Ahmadi, J., and Baratpour, S. (2014), Non-parametric prediction intervals for the lifetime of coherent systems. Statistical Papers, 55, 1019-1034.

Chivers, C. (2012), MH adaptive: General Markov Chain Monte Carlo for Bayesian Inference using adaptive Metropolis-Hastings sampling, R package.

Kaminsky, K. S., and Rhodin, L. S. (1985), Maximum likelihood prediction. Annals of the Institute of Statistical Mathematics, 37, 507-517.

Kochar, S., Mukerjee, H., and Samaniego, F. J. (1999), The Signature of a Coherent System and its Application to Comparisons Among Systems. Naval Research Logistics, 46, 507-523.

Navarro, J., and R. Rubio, R. (2010), Computations of coherent systems with five components. Communications in Statistics - Simulation and Computation, 39, 68-84.

Navarro, J., Ruiz, J. M., and Sandoval, C. J. (2007), Properties of coherent systems with dependent components, Communications in Statistics - Theory and Methods, 36, 175-191.

Navarro, J., Samaniego, F. J., Balakrishnan, N., and Bhattacharya, D. (2008), On the application and extension of system signatures in engineering reliability. Naval Research Logistics, 55, 313-327.

Ng, H. K. T., Navarro, J., and Balakrishnan, N. (2012), Parametric inference from system lifetime data under a proportional hazard rate model. Metrika, 75, 367-388.

Raqab, M. Z., and Nagaraja, H. N. (1995), On some predictors of future order statistics. Metron, 53, 185-204.

Raqab, M. Z., Alkhalfan, L. A., Bdair, O. M., and Balakrishnan, N. (2019), Maximum likelihood prediction of records from 3-parameter Weibull distribution and some approximations. Journal of Computational and Applied Mathematics, 356, 118-132. 
R Core Team (2019), R: A Language and Environment for Statistical Computing. R Foundation for Statistical Computing, Vienna, Austria.

Robert, C., and Casella, G. (2004), Monte Carlo Statistical Methods, 2nd edition. New York: Springer.

Saadati Nik, A., Asgharzadeh, A., and Raqab, M. Z. (2020), Prediction Methods for Future Failure Times Based on Type-II Right-Censored Samples from New ParetoType Distribution. Journal of Statistical Theory and Practice, 14, 39.

Samaniego, F. J. (1985), On closure of the IFR class under formation of coherent systems. IEEE Transactions on Reliability Theory, 34, 69-72.

Samaniego, F. J. (2007), System Signatures and their Applications in Engineering Reliability, International Series in Operations Research and Management Science 110. Springer, New York.

Yang, Y., Ng, H. K. T., and Balakrishnan, N. (2016), A stochastic expectationmaximization algorithm for the analysis of system lifetime data with known signature, Computational Statistics, 31, 609-641.

Yang, Y., Ng, H. K. T., and Balakrishnan, N. (2019), Expectation maximization algorithm for system based lifetime data with unknown system structure. AStA Advanced Statistical Analysis, 103, 69-98.

Zhang, J., Ng, H. K. T., and Balakrishnan, N. (2015a), Statistical inference of component lifetimes with location-scale distributions from censored system failure data with known signature. IEEE Transactions on Reliability, 64, 613-626.

Zhang, J., Ng, H. K. T., and Balakrishnan, N. (2015b), Tests for homogeneity of distributions of component lifetimes from system lifetime data with known system signatures. Naval Research Logistics, 62, 550-563. 